\title{
Volume minimization and conformally Kähler, Einstein-Maxwell geometry
}

\author{
By Akito FutAKI and Hajime Ono
}

(Received Apr. 20, 2017)

\begin{abstract}
Let $M$ be a compact complex manifold admitting a Kähler structure. A conformally Kähler, Einstein-Maxwell metric (cKEM metric for short) is a Hermitian metric $\tilde{g}$ on $M$ with constant scalar curvature such that there is a positive smooth function $f$ with $g=f^{2} \tilde{g}$ being a Kähler metric and $f$ being a Killing Hamiltonian potential with respect to $g$. Fixing a Kähler class, we characterize such Killing vector fields whose Hamiltonian function $f$ with respect to some Kähler metric $g$ in the fixed Kähler class gives a cKEM metric $\tilde{g}=f^{-2} g$. The characterization is described in terms of critical points of certain volume functional. The conceptual idea is similar to the cases of Kähler-Ricci solitons and Sasaki-Einstein metrics in that the derivative of the volume functional gives rise to a natural obstruction to the existence of cKEM metrics. However, unlike the Kähler-Ricci soliton case and Sasaki-Einstein case, the functional is neither convex nor proper in general, and often has more than one critical points. The last observation matches well with the ambitoric examples studied earlier by LeBrun and Apostolov-Maschler.
\end{abstract}

\section{Introduction.}

Let $(M, J)$ be a compact Kähler manifold. We call a Hermitian metric $\tilde{g}$ on $(M, J)$ a conformally Kähler, Einstein-Maxwell metric (cKEM metric for short) if it satisfies the following three conditions:

(a) There exists a positive smooth function $f$ on $M$ such that $g=f^{2} \tilde{g}$ is Kähler.

(b) The Hamiltonian vector field $K=\operatorname{Jgrad}_{g} f$ is Killing for both $g$ and $\tilde{g}$.

(c) The scalar curvature $s_{\tilde{g}}$ of $\tilde{g}$ is constant.

Since the Ricci tensors $\operatorname{Ric}_{g}$ and $\operatorname{Ric}_{\tilde{g}}$ of $g$ and $\tilde{g}$ are related by

$$
\operatorname{Ric}_{\tilde{g} 0}=\operatorname{Ric}_{g 0}+2 f^{-1} \operatorname{Hess}_{0} f
$$

where [ $]_{0}$ denotes (throughout this paper) the trace free part (c.f. (1.161b) in Besse $[\mathbf{5}]$ ), the condition (b) is equivalent to

$\left(\mathrm{b}^{\prime}\right) \operatorname{Ric}_{\tilde{g}}(J \cdot, J \cdot)=\operatorname{Ric}_{\tilde{g}}(\cdot, \cdot)$.

The condition (c) is equivalent to

2010 Mathematics Subject Classification. Primary 53C55; Secondary 53C25.

Key Words and Phrases. conformally Kähler Einstein-Maxwell metric, volume minimization. 


$$
s_{\tilde{g}}=2\left(\frac{2 m-1}{m-1}\right) f^{m+1} \Delta_{g}\left(\frac{1}{f}\right)^{m-1}+s_{g} f^{2}=\text { const },
$$

where $\operatorname{dim}_{\mathbf{C}} M=m$.

If $\tilde{g}$ is a cKEM metric and if $\operatorname{dim}_{\mathbf{R}}=4$, then one obtains a solution $(M, h, F)$ of the following Einstein-Maxwell equation studied in General Relativity (see LeBrun [21]):

(i) $h$ is a Riemannian metric. (In our case $h=\tilde{g}$ ).

(ii) $F$ is a real 2 -form.

(iii) $d F=0, d * F=0,[\text { Ric }+F \circ F]_{0}=0$. Here $(F \circ F)_{j k}=F_{j}^{\ell} F_{\ell k}$. (In our case $F^{+}$ is the Kähler form $\omega_{g}$, and $F^{-}=f^{-2} \rho_{0}(\tilde{g}) / 2$ with $\rho_{0}(\tilde{g})$ the traceless Ricci form of $\tilde{g}$.)

Except for the constant scalar curvature Kähler (cscK for short) metrics in which case $f$ is a constant function, not many examples are known. The most well-known examples may be the conformally Kähler Einstein metrics by Page [25] on the one-point-blow-up of $\mathbf{C P}^{2}$, by Chen-LeBrun-Weber [7] on the two-point-blow-up of $\mathbf{C P}^{2}$. Further examples are the ones by Apostolov-Calderbank-Gauduchon [1], [2] on 4-orbifolds and by BérardBergery $[\mathbf{4}]$ on $\mathbf{P}^{1}$-bundles over Fano Kähler-Einstein manifolds. In the more recent studies, non-Einstein cKEM examples are constructed by LeBrun [21], [22] showing that there are ambitoric examples on $\mathbf{C P}^{1} \times \mathbf{C P}^{1}$ and the one-point-blow-up of $\mathbf{C P}^{2}$, and by Koca-Tønnesen-Friedman $[\mathbf{1 8}]$ on ruled surfaces of higher genus.

In [3], Apostolov and Maschler initiated a study in the framework similar to the Kähler geometry, and set the existence problem of cKEM metrics in the DonaldsonFujiki picture [9], [12]. In particular, fixing a Kähler class, they defined an obstruction to the existence of cKEM metrics in a similar manner to the Kähler-Einstein and cscK cases $[\mathbf{1 3}],[\mathbf{1 4}]$. They further studied the toric surfaces and showed the equivalence between the existence of cKEM metrics and toric K-stability on toric surfaces with convex quadrilateral moment map images, extending earlier works by Legendre [23] and Donaldson [10], [11]. We remark that Lichnerowicz-Matsushima reductiveness theorem for cscK manifolds is also extended to the cKEM manifolds by Lahdili [19] and us [16] independently.

The purpose of the present paper is to study for which Killing vector field we can find a cKEM metric. We show that, fixing a Kähler class, such Killing vector fields are critical points of certain volume functional. We also show that, for toric manifolds, this idea gives an efficient way to decide which vector fields in the Lie algebra of the torus can have a solution of the cKEM problem. The idea is similar to the cases of Kähler-Ricci solitons and Sasaki-Einstein metrics, so let us digress to these two cases. A KählerRicci soliton is a Kähler metric with its Kähler form $\omega \in c_{1}(M)$ such that there exists a Killing Hamiltonian vector field $X$ in the Lie algebra $\mathfrak{h}$ of the maximal torus of the automorphism group such that

$$
\begin{aligned}
\rho_{\omega} & =\omega+L_{J X} \omega \\
& =\omega+i \partial \bar{\partial} f_{X}
\end{aligned}
$$


where $\rho_{\omega}$ is the Ricci form of $\omega$ and $f_{X}$ is the Hamiltonian function of $X$. To find such $X$ that there is a Kähler form $\omega$ satisfying the Kähler-Ricci soliton equation, let $g$ be an arbitrary Kähler metric with its Kähler class $\omega_{g} \in c_{1}(M)$, and let $h_{g}$ be a smooth function such that

$$
\rho_{g}-\omega_{g}=i \partial \bar{\partial} h_{g}
$$

Tian and Zhu defined in [27] a functional $F_{X}: \mathfrak{h} \rightarrow \mathbf{R}$ by

$$
F_{X}(Y)=\int_{M}(J Y)\left(h_{g}-f_{X}\right) e^{f_{X}} \omega_{g}^{m}
$$

where $f_{X}$ is the Hamiltonian function of $X$ with the normalization $\int_{M} e^{f_{X}} \omega_{g}^{m}=\int_{M} \omega_{g}^{m}$. $F_{X}$ is independent of the choice of $\omega_{g} \in c_{1}(M)$, and if there exists a Kähler-Ricci soliton for $X$ then $F_{X}$ vanishes identically. To find such $X$ with vanishing $F_{X}$, they considered the weighted volume functional $V: \mathfrak{h} \rightarrow \mathbf{R}$ defined by

$$
V(Z)=\int_{M} e^{u_{Z}} \omega_{g}^{m}
$$

where $u_{Z}$ is the Hamiltonian function of $Z \in \mathfrak{h}$ with the normalization $\int_{M} u_{Z} e^{h_{g}} \omega_{g}^{m}=0$. They showed that $V$ is independent of $\omega_{g}$, that $d V_{X}(Y)=c F_{X}(Y)$ with a constant $c$, that $V$ is a strictly convex proper function, and that there is a unique minimum $X$. This minimum $X$ is the right choice to solve the Kähler-Ricci soliton equation.

Let us turn to the Sasaki-Einstein metrics. An odd dimensional Riemannian manifold $S$ is said to be a Sasakian manifold if its Riemannian cone manifold $C(S)$ is a Kähler manifold, and a Sasakian manifold $S$ is said to be a Sasaki-Einstein manifold if $S$ is also an Einstein manifold. A fundamental fact is that $S$ is Sasaki-Einstein if and only if its cone $C(S)$ is a Ricci-flat Kähler manifold, and also if and only if the local leaf spaces of the 1-dimensional foliation generated by the Reeb vector field $J(r \partial / \partial r)$ have KählerEinstein metrics where $r$ denotes the radial coordinate on the cone $C(S)$. There is an obstruction to the existence of Sasaki-Einstein metrics similar to Kähler-Einstein metrics [17], [6]. Fixing a holomorphic structure of the cone $C(S)$, a natural deformation space of Sasakian structures is the deformation space of the cone structures of $C(S)$. If such a deformation is given by $r \mapsto r^{\prime}=r e^{\varphi}$ then we have a deformation of the Reeb vector field $J(r \partial / \partial r) \mapsto J\left(r^{\prime} \partial / \partial r^{\prime}\right)$. Thus, this deformation can be regarded as a deformation of the Reeb vector fields. Let us define the volume functional $V: \operatorname{KCS}(C(S), J) \rightarrow \mathbf{R}$ on the space $\operatorname{KCS}(C(S), J)$ of the Kähler cone structures with a fixed holomorphic structure $J$ by $V(S, g)=\operatorname{vol}(S, g)$ where $S=\{r=1\}$ is the Sasakian manifold determined by the Kähler cone structure. Denote by $G$ the maximal torus of the group of automorphisms commuting with the flow generated by $r \partial / \partial r$. Martelli-Sparks-Yau [24] showed that the derivative $d V_{(S, g)}$ gives rise to a linear function on $\operatorname{Lie}(G)$ which coincides with the obstruction to the existence of Sasaki-Einstein metrics mentioned above. They further showed that, when $S$ is toric (meaning $C(S)$ is toric), the volume functional $V$ restricted to the space of toric deformations (meaning deformations of the Reeb vector field in the Lie algebra of the torus) is a strictly convex proper function, and the unique minimum 
is the right choice of the Reeb vector field, i.e. the right choice of the Sasakian structure to solve the Sasaki-Einstein equation because this minimum assures the vanishing of the obstruction. For this choice we can always find a Sasaki-Einstein metric $[\mathbf{1 7}],[\mathbf{8}]$. See also the survey articles $[\mathbf{1 5}],[\mathbf{2 6}]$.

To be more precise in our cKEM problem, let $G$ be a maximal torus of a maximal reductive subgroup of the automorphism group, and take $K \in \mathfrak{g}:=\operatorname{Lie}(G)$. Let $\omega_{0}$ be a Kähler form, and $\Omega=\left[\omega_{0}\right] \in H_{\mathrm{DR}}^{2}(M, \mathbf{R})$ be a fixed Kähler class. The problem is to find a $G$-invariant Kähler metric $g$ with its Kähler form $\omega_{g} \in \Omega$ such that

(i) $\tilde{g}=f^{-2} g$ is a cKEM metric,

(ii) $\operatorname{Jgrad}_{g} f=K$.

Denote by $\mathcal{K}_{\Omega}^{G}$ the space of $G$-invariant Kähler metrics $g$ with $\omega_{g} \in \Omega$. For any $(K, a, g) \in$ $\mathfrak{g} \times \mathbf{R} \times \mathcal{K}_{\Omega}^{G}$, there exists a unique function $f_{K, a, g} \in C^{\infty}(M, \mathbf{R})$ satisfying the following two conditions:

$$
\iota_{K} \omega_{g}=-d f_{K, a, g}, \quad \int_{M} f_{K, a, g} \frac{\omega_{g}^{m}}{m !}=a .
$$

Noting $\min \left\{f_{K, a, g} \mid x \in M\right\}$ is independent of $g$ with $\omega_{g} \in \Omega$ (see Section 2), we put

$$
\begin{aligned}
& \mathcal{P}_{\Omega}^{G}:=\left\{(K, a) \in \mathfrak{g} \times \mathbf{R} \mid f_{K, a, g}>0\right\}, \\
& \mathcal{H}_{\Omega}^{G}:=\left\{\tilde{g}_{K, a}=\frac{1}{f_{K, a, g}^{2}} g \mid(K, a) \in \mathcal{P}_{\Omega}^{G}, g \in \mathcal{K}_{\Omega}^{G}\right\} .
\end{aligned}
$$

Hereafter the Kähler metric $g$ and its Kähler form $\omega_{g}$ are often identified, and $\omega_{g}$ is often denoted by $\omega$. Fixing $(K, a) \in P_{\Omega}^{G}$, put

$$
\mathcal{H}_{\Omega, K, a}^{G}:=\left\{\tilde{g}_{K, a} \mid g \in \mathcal{K}_{\Omega}^{G}\right\}
$$

and

$$
d_{\Omega, K, a}:=\frac{S\left(\tilde{g}_{K, a}\right)}{\operatorname{Vol}\left(\tilde{g}_{K, a}\right)}=\frac{\int_{M} s_{\tilde{g}_{K, a}}\left(\frac{1}{f_{K, a, g}}\right)^{2 m} \frac{\omega^{m}}{m !}}{\int_{M}\left(\frac{1}{f_{K, a, g}}\right)^{2 m} \frac{\omega^{m}}{m !}} .
$$

Then $d_{\Omega, K, a}$ is a constant independent of the choice of $g \in \mathcal{K}_{\Omega}^{G}$ as shown in $[\mathbf{3}]$. For any real number $\gamma$, let us put further

$$
\tilde{\mathcal{P}}_{\Omega}^{G}:=\left\{(K, a) \in \mathcal{P}_{\Omega}^{G} \mid d_{\Omega, K, a}=\gamma\right\} .
$$

We may normalize $\gamma=1,0$ or -1 if it is more convenient, but this is not really necessary.

The main result in this paper is the following volume minimization property of cKEM metrics.

TheOREM 1.1. Let $(K, a) \in \tilde{\mathcal{P}}_{\Omega}^{G}$. Then if there exists a conformally Kähler, Einstein-Maxwell metric $\tilde{g}_{K, a} \in \mathcal{H}_{\Omega, K, a}^{G}$ then $(K, a)$ is a critical point of $\mathrm{Vol}: \tilde{\mathcal{P}}_{\Omega}^{G} \rightarrow \mathbf{R}$ 
given by $\operatorname{Vol}(K, a):=\operatorname{Vol}\left(\tilde{g}_{K, a}\right)$ for $(K, a) \in \tilde{\mathcal{P}}_{\Omega}^{G}$. Further, $(K, a)$ is a critical point of $\mathrm{Vol}: \tilde{\mathcal{P}}_{\Omega}^{G} \rightarrow \mathbf{R}$ if and only if $\mathfrak{F}_{\Omega, K, a}^{G} \equiv 0$.

Here $\mathfrak{F}_{\Omega, K, a}^{G}$ is an obstruction to the existence of cKEM metric defined in [3], described as follows. For $(K, a) \in \mathcal{P}_{\Omega}^{G}$, the following hold:

$$
c_{\Omega, K, a}:=\frac{\int_{M} s_{\tilde{g}_{K, a}}\left(\frac{1}{f_{K, a, g}}\right)^{2 m+1} \frac{\omega^{m}}{m !}}{\int_{M}\left(\frac{1}{f_{K, a, g}}\right)^{2 m+1} \frac{\omega^{m}}{m !}}
$$

is a constant independent of the choice of $g \in \mathcal{K}_{\Omega}^{G}$. Then

$$
\mathfrak{F}_{\Omega, K, a}^{G}: \mathfrak{g} \rightarrow \mathbf{R}, \quad \mathfrak{F}_{\Omega, K, a}^{G}(H):=\int_{M}\left(\frac{s_{\tilde{g}_{K, a}}-c_{\Omega, K, a}}{f_{K, a, g}^{2 m+1}}\right) f_{H, b, g} \frac{\omega^{m}}{m !}
$$

is a linear function independent of the choice of $(g, b) \in \mathcal{K}_{\Omega}^{G} \times \mathbf{R}$. Obviously, if there exists a constant scalar curvature metric in $\mathcal{H}_{\Omega, K, a}$, then $\mathfrak{F}_{\Omega, K, a}^{G}$ is identically zero. For terminological convenience we call $\mathfrak{F}_{\Omega, K, a}^{G}$ the cKEM-Futaki invariant. A merit of Theorem 1.1 is to give a systematic computation of the cKEM-Futaki invariant.

This paper is organized as follows. In Section 2 we give a proof of Theorem 1.1. In Section 3 we give examples of non Kähler cKEM metrics. This is an extension of LeBrun's construction $([\mathbf{2 1}])$ on $\mathbf{C P}^{1} \times \mathbf{C P}^{1}$ to $\mathbf{C P}^{1} \times M$ for higher dimensional $M$ 's. In Section 4, we use Maxima (a descendant of Macsyma) to compute the cKEM-Futaki invariant of $\mathbf{C P} \mathbf{P}^{1} \times \mathbf{C} \mathbf{P}^{1}$, the blow-up of $\mathbf{C P}^{2}$ at one point and other Hirzebruch surfaces.

\section{Proof of Theorem 1.1.}

Let $M$ be a compact $n$-manifold with $n \geq 3$. Let $\operatorname{Riem}(M)$ denote the set of all Riemannian metrics on $M, \operatorname{Ric}_{g}$ the Ricci tensor of $g, s_{g}$ the scalar curvature of $g$, and $d v_{g}$ the volume form of $g$. The normalized Einstein-Hilbert functional $E H: \operatorname{Riem}(M) \rightarrow \mathbf{R}$ is defined by

$$
E H(g):=\frac{S(g)}{(\operatorname{Vol}(g))^{(n-2) / n}}
$$

where $S(g)=\int_{M} s_{g} d v_{g}$ is the total scalar curvature and $\operatorname{Vol}(g)=\int_{M} d v_{g}$ is the volume of $g$.

The following first variation formulae are standard, and can be found in [5]. Let $g_{t}$ be a smooth family of Riemannian metrics such that $g_{0}=g$ and $d / d t_{\mid t=0} g_{t}=h$. Then

$$
\left.\frac{d}{d t}\right|_{t=0} S\left(g_{t}\right)=\int_{M}\left\langle\frac{s_{g}}{2} g-\operatorname{Ric}_{g}, h\right\rangle_{g} d v_{g} .
$$

Let $f_{t}$ be a smooth family of positive functions such that $f_{0}=1, d / d t_{\mid t=0} f_{t}=\phi$. Then 


$$
\frac{d}{d t}_{\mid t=0} S\left(f_{t} g\right)=\int_{M}\left\langle\frac{s_{g}}{2} g-\operatorname{Ric}_{g}, \phi g\right\rangle d v_{g}=\frac{n-2}{2} \int_{M} s_{g} \phi d v_{g}
$$

and

$$
\frac{d}{d t}_{\mid t=0} \operatorname{Vol}\left(f_{t} g\right)=\frac{d}{d t} \int_{\mid t=0} \int_{M} f_{t}^{n / 2} d v_{g}=\frac{n}{2} \int_{M} \phi d v_{g}
$$

Therefore

$$
\frac{d}{d t}{ }_{\mid t=0} E H\left(f_{t} g\right)=\frac{n-2}{2(\operatorname{Vol}(g))^{(n-2) / n}} \int_{M}\left(s_{g}-\frac{S(g)}{\operatorname{Vol}(g)}\right) \phi d v_{g} .
$$

By (11), if $s_{g}$ is a constant then $g$ is a critical point of $E H$ restricted to the conformal class of $g$.

Let $f_{t}$ be a smooth family of positive functions such that $f_{0}=f, d / d t_{\mid t=0} f_{t}=\phi$. Denote $\left(1 / f_{t}^{2}\right) g=: \tilde{g}_{t}, \tilde{g}=: \tilde{g}_{0}$. Then

$$
\frac{d}{d t}_{\mid t=0} S\left(\tilde{g}_{t}\right)=\int_{M}\left\langle\frac{s_{\tilde{g}}}{2}-\operatorname{Ric}_{\tilde{g}}, \frac{-2 \phi}{f} \tilde{g}\right\rangle_{\tilde{g}} d v_{\tilde{g}}=(2-n) \int_{M} \frac{s_{\tilde{g}} \phi}{f^{n+1}} d v_{g}
$$

and

$$
\left.\frac{d}{d t}\right|_{t=0} \operatorname{Vol}\left(\tilde{g}_{t}\right)=\left.\frac{d}{d t}\right|_{t=0} \int_{M} \frac{1}{f_{t}^{n}} d v_{g}=-n \int_{M} \frac{\phi}{f^{n+1}} d v_{g}
$$

Therefore

$$
\frac{d}{d t}{ }_{t=0} E H\left(\tilde{g}_{t}\right)=\frac{2-n}{(\operatorname{Vol}(\tilde{g}))^{(n-2) / n}} \int_{M} \frac{s_{\tilde{g}}-S(\tilde{g}) / \operatorname{Vol}(\tilde{g})}{f^{n+1}} \phi d v_{g}
$$

We wish to apply the formula (12) to the existence problem of cKEM metrics. Let us recall the situation explained in the introduction. Let $M$ be a compact Kähler manifold of complex dimension $m$ so that $n=2 m$. Fix a compact group $G \subset \operatorname{Aut}_{r}(M, J)$ in the group of reduced automorphisms of $(M, J)$, and consider a fixed Kähler class $\Omega$ on $(M, J)$. Denote by $\mathcal{K}_{\Omega}^{G}$ the space of $G$-invariant Kähler metrics $\omega$ in $\Omega$. For any $(K, a, g) \in \mathfrak{g} \times \mathbf{R} \times \mathcal{K}_{\Omega}^{G}$, there exists unique function $f_{K, a, g} \in C^{\infty}(M, \mathbf{R})$ satisfying the following two conditions:

$$
\iota_{K} \omega=-d f_{K, a, g}, \quad \int_{M} f_{K, a, g} \frac{\omega^{m}}{m !}=a .
$$

By (12) and (13), it is easy to see that $f_{K, a, g}$ has the following properties:

$$
\begin{gathered}
f_{K+H, a+b, g}=f_{K, a, g}+f_{H, b, g}, \\
f_{0, a, g}=\frac{a}{\operatorname{Vol}(M, \omega)}, \\
f_{C K, C a, g}=C f_{K, a, g} .
\end{gathered}
$$

If $K \neq 0$, 


$$
m_{K, \Omega}:=\min \left\{f_{K, 0, g} \mid x \in M\right\}<0 .
$$

Note here that, by Lemma 1 of $[\mathbf{3}], \min \left\{f_{K, 0, g} \mid x \in M\right\}$ is independent of the choice of $g \in \mathcal{K}_{\Omega}^{G}$. Hence $f_{K, a, g}$ is positive if $a>l_{K, \Omega}:=-m_{K, \Omega} \operatorname{Vol}(M, \omega)$, by (14) and (15).

The set $\mathcal{P}_{\Omega}^{G}$ defined in (3) in the introduction then can be expressed as

$$
\mathcal{P}_{\Omega}^{G}=\left\{(K, a) \in \mathfrak{g} \times \mathbf{R} \mid a>l_{K, \Omega}\right\} .
$$

With the notation of $\mathcal{H}_{\Omega, K, a}^{G}$ given in (5), the main problem of the cKEM metrics we wish to consider is the following :

Problem 2.1. Is there a cKEM metric in $\mathcal{H}_{\Omega, K, a}^{G}$ for $(K, a) \in \mathcal{P}_{\Omega}^{G}$ ?

By (13), the Hamiltonian function $f_{K, a, g}$ of $K$ with respect to the Kähler metric $g$ is Killing for both $g$ and $\tilde{g}_{K, a}=f_{K, a, g}^{-2} g$. So the problem above is equivalent to the existence of the constant scalar curvature metric in $\mathcal{H}_{\Omega, K, a}^{G}$. As was explained in the introduction, Apostolov and Maschler [3] introduced an obstruction $\mathfrak{F}_{\Omega, K, a}^{G}: \mathfrak{g} \rightarrow \mathbf{R}$ to this problem, see (9).

Fix $(K, a) \in \mathcal{P}_{\Omega}^{G}$. By Corollary 2 of [3], the functional $S$ is constant on $\mathcal{H}_{\Omega, K, a}^{G}$. Similarly by the proof of Lemma 1 of [3], Vol is constant on $\mathcal{H}_{\Omega, K, a}^{G}$. Thus we obtain the following proposition.

Proposition 2.2. With the notations as above, we have the following.

1. $\operatorname{For}(K, a) \in \mathcal{P}_{\Omega}^{G}$,

$$
d_{\Omega, K, a}:=\frac{S\left(\tilde{g}_{K, a}\right)}{\operatorname{Vol}\left(\tilde{g}_{K, a}\right)}=\frac{\int_{M} s_{\tilde{g}_{K, a}}\left(\frac{1}{f_{K, a, g}}\right)^{2 m} \frac{\omega^{m}}{m !}}{\int_{M}\left(\frac{1}{f_{K, a, g}}\right)^{2 m} \frac{\omega^{m}}{m !}}
$$

is a constant independent of the choice of $g \in \mathcal{K}_{\Omega}^{G}$.

2. The function

$$
V: \mathcal{P}_{\Omega}^{G} \rightarrow \mathbf{R}, \quad(K, a) \mapsto E H\left(\tilde{g}_{K, a}\right)
$$

is well-defined.

THEOREM 2.3. We have the following results.

(a) If there exists a cKEM metric $\tilde{g}_{K, a} \in \mathcal{H}_{\Omega, K, a}^{G}$, then $(K, a)$ is a critical point of $V: \mathcal{P}_{\Omega}^{G} \rightarrow \mathbf{R}$

(b) If $(K, a)$ is a critical point of $V: \mathcal{P}_{\Omega}^{G} \rightarrow \mathbf{R}, \mathfrak{F}_{\Omega, K, a}^{G}$ vanishes identically.

Proof. The first statement (a) is trivial by the first variation of the EinsteinHilbert functional (12). To prove the second statement (b), suppose that $(K, a) \in \mathcal{P}_{\Omega}^{G}$ is 
a critical point of $V$. For $H \in \mathfrak{g}$,

$$
\begin{aligned}
0 & =\left.\frac{d}{d t}\right|_{t=0} V(K+t H, a) \\
& =\frac{2-2 m}{\operatorname{Vol}\left(M, \tilde{g}_{K, a}\right)^{(m-1) / m}} \int_{M}\left(\frac{s_{\tilde{g}_{K, a}}-d_{\Omega, K, a}}{f_{K, a, g}^{2 m+1}}\right) f_{H, 0, g} \frac{\omega^{m}}{m !}
\end{aligned}
$$

by (12), (14) and (16). Note here that, in general, $c_{\Omega, K, a}$ does not coincide with $d_{\Omega, K, a}$. So (19) does not mean the vanishing of cKEM-Futaki invariant $\mathfrak{F}_{\Omega, K, a}^{G}$ in (9). However if there exists a cKEM metric in $\mathcal{H}_{\Omega, K, a}, c_{\Omega, K, a}=d_{\Omega, K, a}$ holds, i.e. $c_{\Omega, K, a}-d_{\Omega, K, a}$ is an obstruction to the existence of cKEM metric. This obstruction can be represented as the $\mathbf{R}$-direction first variation of $V$ as follows:

$$
\begin{aligned}
0 & =\frac{d}{d t}_{\mid t=0} V(K, a+t) \\
& =\frac{2-2 m}{\operatorname{Vol}\left(M, \tilde{g}_{K, a}\right)^{(2 m-1) / m}}\left(c_{\Omega, K, a}-d_{\Omega, K, a}\right) \int_{M} \frac{1}{f_{K, a, g}^{2 m+1}} \frac{\omega^{m}}{m !} .
\end{aligned}
$$

Therefore if $(K, a) \in \mathcal{P}_{\Omega}^{G}$ is a critical point of $V$, then $\mathfrak{F}_{\Omega, K, a}^{G} \equiv 0$.

There exists a cKEM metric in $\mathcal{H}_{\Omega, K, a}^{G}$ if and only if there exists a cKEM metric in $\mathcal{H}_{\Omega, C K, C a}^{G}$ for $C>0$. Therefore Problem 2.1 is equivalent to the following problem.

Problem 2.4. Is there a cKEM metric in $\mathcal{H}_{\Omega, K, a}^{G}$ for $[(K, a)] \in \mathcal{P}_{\Omega}^{G} / \mathbf{R}_{>0}$ ?

So we wish to have good representatives of elements in $\mathcal{P}_{\Omega}^{G} / \mathbf{R}_{>0}$. This is the motivation to define $\tilde{\mathcal{P}}_{\Omega}^{G}$ as in the introduction, see (7). Since $d_{\Omega, C K, C a}=C^{2} d_{\Omega, K, a}$ for $C>0$,

$$
\tilde{\mathcal{P}}_{\Omega}^{G} \simeq \mathcal{P}_{\Omega}^{G} / \mathbf{R}_{>0}, \quad(K, a) \mapsto[(K, a)]
$$

is bijective.

Proof of Theorem 1.1. Let $(K(t), a(t)), t \in(-\varepsilon, \varepsilon)$ be a smooth curve in $\tilde{\mathcal{P}}_{\Omega}^{G}$ such that $(K(0), a(0))=(K, a),\left(K^{\prime}(0), a^{\prime}(0)\right)=(H, b)$. Then

$$
S\left(\tilde{g}_{K(t), a(t)}\right)=\gamma \operatorname{Vol}\left(\tilde{g}_{K(t), a(t)}\right)
$$

holds for any $t \in(-\varepsilon, \varepsilon)$. By differentiating this equation at $t=0$, we have

$$
(m-1) \int_{M} \frac{s_{\tilde{g}_{K, a}} f_{H, b, g}}{f_{K, a, g}^{2 m+1}} \frac{\omega^{m}}{m !}=m \gamma \int_{M} \frac{f_{H, b, g}}{f_{K, a, g}^{2 m+1}} \frac{\omega^{m}}{m !} .
$$

Hence

$$
\mathfrak{F}_{\Omega, K, a}^{G}(H)=\left(\frac{m \gamma}{m-1}-c_{\Omega, K, a}\right) \int_{M} \frac{f_{H, b, g}}{f_{K, a, g}^{2 m+1}} \frac{\omega^{m}}{m !}
$$




$$
=\left.\left(\frac{\gamma}{m-1}-\frac{c_{\Omega, K, a}}{m}\right) \frac{d}{d t}\right|_{t=0} \operatorname{Vol}\left(M, \tilde{g}_{K(t), a(t)}\right)
$$

holds by (9) and (21). If there exists a cKEM metric in $\mathcal{H}_{\Omega, K, a}^{G}$ for $(K, a) \in \tilde{\mathcal{P}}_{\Omega}^{G}$, then

$$
c_{\Omega, K, a}=d_{\Omega, K, a}=\gamma
$$

and

$$
\mathfrak{F}_{\Omega, K, a}^{G}(H)=0 .
$$

Therefore for any nonzero real constant $\gamma$ we have

$$
\left.\frac{d}{d t}\right|_{\mid t=0} \operatorname{Vol}\left(M, \tilde{g}_{K(t), a(t)}\right)=0 .
$$

If $\gamma=0$ then the left hand side of (21) vanishes. From this and the equalities in (19) and (20) we obtain (23).

\section{Examples of non Kähler cKEM metrics.}

In this section, we construct compact, non-Kähler examples of cKEM metrics of dimension greater than two.

Let $g_{1}$ be an $S^{1}$-invariant metric on $\mathbf{C P}^{1}$ and $g_{2}$ a Kähler metric with $s_{g_{2}}=c$ on an $(m-1)$-dimensional compact complex manifold $M$. The $S^{1}$-invariant metric $g_{1}$ can be written in the action-angle coordinates $(t, \theta) \in(a, b) \times(0,2 \pi]$ as

$$
g_{1}=\frac{d t^{2}}{\Psi(t)}+\Psi(t) d \theta^{2}
$$

for some smooth function $\Psi(t)$. The Hamiltonian function of the generator of the $S^{1}$ action is $t^{1}$. Suppose that $a>0$. We will look for $\Psi$ such that the Hermitian metric $g / t^{2}$, where $g=g_{1}+g_{2}$, on $\mathbf{C P}^{1} \times M$ has constant scalar curvature. Since $\partial / \partial \theta$ is Killing both for $g$ and $g / t^{2}$, if we find such $\Psi, g / t^{2}$ is a non-Kähler cKEM metric. The scalar curvature of $g_{1}$ is given by

$$
s_{1}=\Delta_{g_{1}} \log \Psi=-\Psi^{\prime \prime}(t) .
$$

Thus the scalar curvature of $g$ is given by

$$
s=s_{1}+s_{2}=c-\Psi^{\prime \prime}(t) .
$$

We want to arrange that the scalar curvature of $h=t^{-2} g$ is $d$, which is to say that

$$
d=s_{h}=2\left(\frac{2 m-1}{m-1}\right) t^{m+1} \Delta_{g}\left(t^{1-m}\right)+\left(c-\Psi^{\prime \prime}(t)\right) t^{2} .
$$

\footnotetext{
${ }^{1}$ In this section, instead of sliding the Hamiltonian function by constant, we move the interval $(a, b)$ (moment image).
} 
We may rewrite this as

$$
c-\Psi^{\prime \prime}=\frac{d}{t^{2}}-2\left(\frac{2 m-1}{m-1}\right) t^{m-1} \Delta_{g_{1}}\left(t^{1-m}\right)
$$

since the Hessian of $t$ is trivial in the $M$-directions. Since

$$
\Delta_{g_{1}}\left(\frac{1}{t^{m-1}}\right)=(m-1)\left(\frac{\Psi}{t^{m}}\right)^{\prime}
$$

the equation (24) reduces to the ODE

$$
c-\Psi^{\prime \prime}=\frac{d}{t^{2}}-2(2 m-1) \frac{\Psi^{\prime}}{t}+2 m(2 m-1) \frac{\Psi}{t^{2}},
$$

or equivalently

$$
t^{2} \Psi^{\prime \prime}-2(2 m-1) t \Psi^{\prime}+2 m(2 m-1) \Psi=c t^{2}-d .
$$

The general solution of the equation (25) is

$$
\Psi(t)=A t^{2 m}+B t^{2 m-1}+\frac{c}{2(m-1)(2 m-3)} t^{2}-\frac{d}{2 m(2 m-1)} .
$$

Now, in order to get a metric on $S^{2}$, we need to impose the boundary conditions that

$$
\Psi(a)=\Psi(b)=0, \Psi^{\prime}(a)=-\Psi^{\prime}(b)=2, \quad \Psi(t)>0(\text { on }(a, b)) .
$$

The first four conditions reduce to a simultaneous linear equation for $A, B, c$ and $d$. The solution is

$$
\begin{aligned}
& A_{a, b, m}=\left(a ^ { 2 } b ^ { 2 } ( a + b ) \left\{2(a-b)\left(b^{2 m-2}+a^{2 m-2}\right) m\right.\right. \\
& \left.\left.+3 b^{2 m-1}-a b^{2 m-2}+a^{2 m-2} b-3 a^{2 m-1}\right\}\right) / E_{a, b, m}, \\
& B_{a, b, m}=\left(2 a ^ { 2 } b ^ { 2 } ( a + b ) \left\{\left((b-a)\left(b^{2 m-1}+a^{2 m-1}\right)\right) m\right.\right. \\
& \left.\left.-\left(b^{m}-a^{m}\right)\left(b^{m}+a^{m}\right)\right\}\right) / E_{a, b, m}, \\
& \frac{c_{a, b, m}}{2(m-1)(2 m-3)}=\left(b^{2 m}\left(2 a^{2 m} b^{2}-2 a^{2 m+2}\right) m\right. \\
& \left.-a^{2} b^{4 m}+b^{2 m}\left(a^{2 m+2}-a^{2 m} b^{2}\right)+a^{4 m} b^{2}\right) / E_{a, b, m}, \\
& \frac{d_{a, b, m}}{2 m(2 m-1)}=\left(b^{2 m}\left(2 a^{2 m+1} b^{3}-2 a^{2 m+3} b\right) m\right. \\
& \left.-a^{4} b^{4 m}+a^{4 m} b^{4}+b^{2 m}\left(2 a^{2 m+3} b-2 a^{2 m+1} b^{3}\right)\right) / E_{a, b, m},
\end{aligned}
$$

where

$$
\begin{aligned}
E_{a, b, m}= & \left(2 a^{2 m} b^{2 m}(b-a)^{2}(b+a)\right) m^{2}-3 a^{2 m} b^{2 m}(b-a)^{2}(b+a) m \\
& -\left(b^{m}-a^{m}\right)\left(b^{m}+a^{m}\right)\left(a^{3} b^{2 m}-a^{2 m} b^{3}\right) .
\end{aligned}
$$


If we set

$$
\Psi_{a, b, m}(t)=A_{a, b, m} t^{2 m}+B_{a, b, m} t^{2 m-1}+\frac{c_{a, b, m}}{2(m-1)(2 m-3)} t^{2}-\frac{d_{a, b, m}}{2 m(2 m-1)},
$$

we have $\Psi_{a, b, m}>0$ on $(a, b)$ by the following lemma for any $m$ and $0<a<b$. Therefore, for $m \geq 2$,

$$
g_{a, b, m}=\frac{d t^{2}}{\Psi_{a, b, m}(t)}+\Psi_{a, b, m}(t) d \theta^{2}
$$

defines a metric on $\mathbf{C P}^{1}$.

Lemma 3.1. Let $m \geq 2$ be an integer and $0<a<b$. If a real valued function

$$
f(t)=\alpha t^{2 m}+\beta t^{2 m-1}+\gamma t^{2}+\delta
$$

satisfies the boundary conditions

$$
f(a)=f(b)=0, \quad f^{\prime}(a),-f^{\prime}(b)>0,
$$

then $f>0$ on $(a, b)$.

Proof. Suppose that there exists $c \in(a, b)$ such that $f(c) \leq 0$. Then, by the boundary condition, there exist at least three critical points of $f$ in $(a, b)$. On the other hand, since

$$
\begin{gathered}
\frac{f^{\prime}(t)}{t}=2 m \alpha t^{2 m-2}+(2 m-1) \beta t^{2 m-3}+2 \gamma \\
\left(\frac{f^{\prime}(t)}{t}\right)^{\prime}=t^{2 m-4}\{2 m(2 m-2) \alpha t+(2 m-1)(2 m-3) \beta\}
\end{gathered}
$$

$f^{\prime} / t$ has at most two zeros in $(a, b)$. This is a contradiction.

Moreover if $g_{2}$ is a Kähler metric with $s_{g_{2}}=c_{a, b, m}$ on an $(m-1)$-dimensional compact complex manifold $M$,

$$
h_{a, b, m}\left(g_{2}\right)=\frac{1}{t^{2}}\left(g_{a, b, m}+g_{2}\right)
$$

is an $S^{1}$-invariant cKEM metric with $s_{h_{a, b, m}\left(g_{2}\right)}=d_{a, b, m}$ on $\mathbf{C P}^{1} \times M$.

For simplicity, we now put $b=a+1$. Let

$$
\mathcal{C}_{m}=\left\{c_{a, a+1, m} \mid a>0\right\} .
$$

Since

$$
\lim _{a \rightarrow+0} c_{a, a+1, m}=\infty, \quad \lim _{a \rightarrow \infty} c_{a, a+1, m}=8 m-8,
$$

we see that $(8 m-8, \infty) \subset \mathcal{C}_{m}$. Hence we have proved the following. 
TheOREm 3.2. Let $c>8 m-8$. Then there exists $a>0$ such that for any Kähler metric $g_{2}$ with $s_{g_{2}}=c=c_{a, a+1, m}$ on an $(m-1)$-dimensional compact complex manifold $M, h_{a, a+1, m}\left(g_{2}\right)$ is an $S^{1}$-invariant $c K E M$ metric on $\mathbf{C P}^{1} \times M$. On the other hand, if $c \notin \mathcal{C}_{m}$, for any $a>0$ and $g_{2}, h_{a, a+1, m}\left(g_{2}\right)$ is not a $c K E M$ metric.

Note here that, for small $m$, e.g. $m=2,3,4,5$, we can directly confirm that $\mathcal{C}_{m}=$ $(8 m-8, \infty)$. Hence in such cases, $c \notin \mathcal{C}_{m}$ if and only if $c \leq 8 m-8$.

This theorem extends the case when $M=\mathbf{C P}^{1}$ due to LeBrun [21].

\section{Computations in the case of toric surfaces.}

Let $(M, J, g)$ be an $m$-dimensional compact toric Kähler manifold. We denote by $\Delta \subset \mathbf{R}^{m}, u$ and $\mathbf{H}^{u}$ the moment polytope, the symplectic potential and the inverse $\operatorname{Hess}(u)^{-1}$ of the Hessian of $u$ respectively. Then by the equation (22) in [3],

$$
\frac{s_{\tilde{g}_{K, a}}}{f_{K, a, g}^{2 m}}=-f_{K, a, g} \sum_{i, j=1}^{m}\left(\frac{1}{f_{K, a, g}^{2 m-1}} \mathbf{H}_{i j}^{u}\right)_{, i j}
$$

holds. Since $f_{K, a, g}$ is an affine linear function of action coordinates, the equation (30) in [3] implies

$$
\int_{M} \frac{s_{\tilde{g}_{K, a}}}{f_{K, a, g}^{2 m}} \frac{\omega^{m}}{m !}=\frac{2(2 \pi)^{m}}{m !} \int_{\partial \Delta} \frac{1}{f_{K, a, g}^{2 m-2}} d \sigma .
$$

On the other hand

$$
\int_{M} \frac{1}{f_{K, a, g}^{2 m}} \frac{\omega^{m}}{m !}=\frac{(2 \pi)^{m}}{m !} \int_{\Delta} \frac{1}{f_{K, a, g}^{2 m}} d \mu
$$

holds. Therefore $V$ is given by

$$
V(K, a)=\frac{4 \pi}{(m !)^{1 / m}} \frac{\int_{\partial \Delta} \frac{1}{f_{K, a, g}^{2 m-2}} d \sigma}{\left(\int_{\Delta} \frac{1}{f_{K, a, g}^{2 m}} d \mu\right)^{(m-1) / m}} .
$$

In what follows, the coordinates of the moment map image of toric Kähler surfaces will be denoted by $\left(\mu_{1}, \mu_{2}\right)$.

\section{1. $\mathrm{CP}^{2}$ case.}

In this case, up to scale, $\Delta$ is the convex hull of the three points $(0,0),(1,0)$ and $(0,1)$. An affine linear function $f=a \mu_{1}+b \mu_{2}+c$ is positive on $\Delta$ if and only if $c, a+c, b+c>0$.

Since

$$
\begin{aligned}
\int_{\partial \Delta} \frac{d \sigma}{\left(a \mu_{1}+b \mu_{2}+c\right)^{2}} & =\frac{3 c+a+b}{c(a+c)(b+c)}, \\
\int_{\Delta} \frac{d \mu_{1} d \mu_{2}}{\left(a \mu_{1}+b \mu_{2}+c\right)^{4}} & =\frac{3 c^{2}+2(a+b) c+a b}{6 c^{2}(a+c)^{2}(b+c)^{2}},
\end{aligned}
$$


we have

$$
\begin{aligned}
V(a, b, c) & :=2 \sqrt{2} \pi \frac{\int_{\partial \Delta} \frac{1}{\left(a \mu_{1}+b \mu_{2}+c\right)^{2}} d \sigma}{\left(\int_{\Delta} \frac{1}{\left(a \mu_{1}+b \mu_{2}+c\right)^{4}} d \mu_{1} d \mu_{2}\right)^{1 / 2}} \\
& =\frac{4 \sqrt{3} \pi(3 c+a+b)}{\sqrt{3 c^{2}+2(a+b) c+a b}}
\end{aligned}
$$

and

$$
\frac{\partial V}{\partial c}(a, b, c)=-\frac{4 \sqrt{3} \pi\left(a^{2}-a b+b^{2}\right)}{\left(3 c^{2}+2(a+b) c+a b\right)^{3 / 2}} .
$$

Therefore, by (20) and $(31), \mathcal{H}_{\Omega,(a, b), c}^{T^{2}}$ admits cKEM equation only if $(a, b)=(0,0)$. (Note that the notation $\mathcal{H}_{\Omega,(a, b), c}^{T^{2}}$ is a replacement of the previous notation $\mathcal{H}_{\Omega, K, a}^{G}$; this is allowed because $(K, a)$ is determined by $((a, b), c)$.) Hence, we obtain the following result.

Proposition 4.1. Up to constant multiple, the Fubini-Study metric is the only $T^{2}$-invariant cKEM metric on $\mathbf{C P}^{2}$.

Note that it is possible to prove this proposition without using the volume minimization. In fact, (iii) in the introduction can be written as

$$
\operatorname{Ric}_{0}=-F^{+} \circ F^{-}
$$

with $F^{+}$and $F^{-}$are self-dual and anti-self dual harmonic forms (c.f. [21]). Thus, on $\mathbf{C P}^{2}$, we have $F^{-}=0$, and the cKEM metric is Einstein. But compact Hermitian Einstein 4-manifolds are classified by LeBrun [20] to be either the Fubini-Study metric on $\mathbf{C P}^{2}$, the Page metric or Chen-LeBrun-Weber metric. Thus the Fubini-Study metric is the only cKEM metric on $\mathbf{C P}^{2}$. Orbifold cKEM metrics on weighted projective planes are also classified by Apostolov-Maschler [3], Theorem 4.

\section{2. $\mathrm{CP}^{1} \times \mathbf{C P}^{1}$ case.}

Let $\Delta_{p}$ be the convex hull of $(0,0),(p, 0),(p, 1),(0,1)$, where $p \geq 1$. An affine linear function $f=a \mu_{1}+b \mu_{2}+c$ is positive on $\Delta_{p}$ if and only if $c, p a+c, p a+b+c, b+c>0$. We denote

$$
\begin{aligned}
& \mathcal{P}_{p}:=\left\{(a, b, c) \in \mathbf{R}^{3} \mid c, p a+c, p a+b+c, b+c>0\right\}, \\
& \mathcal{P}_{p}(1):=\mathcal{P}_{p} \cap\{(a, b, c) \mid b+p a+2 c=1\} .
\end{aligned}
$$

Note that this choice of $b+p a+2 c=1$ can be replaced by any other affine linear function giving a slice of $\mathcal{P}_{p}$. We chose this simply because it gives a simpler computation. For $(a, b, c) \in \mathcal{P}_{p}$, we define $s_{p}(a, b, c)$ and $v_{p}(a, b, c)$ by 


$$
\begin{aligned}
\int_{\partial \Delta_{p}} & \frac{d \sigma}{\left(a \mu_{1}+b \mu_{2}+c\right)^{2}} \\
& =\frac{1}{c(b+c)}+\frac{p}{(b+c)(b+p a+c)}+\frac{1}{(b+p a+c)(p a+c)}+\frac{p}{(p a+c) c} \\
& =\frac{\left(2 a c+a^{2}+a b\right) p^{2}+\left(2 c^{2}+2(a+b) c+a b+b^{2}\right) p+2 c^{2}+2 b c}{(p a+c)(b+p a+c)(b+c) c} \\
& =\frac{s_{p}(a, b, c)}{(p a+c)(b+p a+c)(b+c) c}
\end{aligned}
$$

and

$$
\begin{aligned}
& \int_{\Delta_{p}} \frac{d \mu_{1} d \mu_{2}}{\left(a \mu_{1}+b \mu_{2}+c\right)^{4}} \\
& =p\left\{\left(2 a^{3} c+a^{3} b\right) p^{3}+\left(8 a^{2} c^{2}+8 a^{2} b c+2 a^{2} b^{2}\right) p^{2}\right. \\
& \quad+\left(12 a c^{3}+18 a b c^{2}+8 a b^{2} c+a b^{3}\right) p \\
& \left.\quad+6 c^{4}+12 b c^{3}+8 b^{2} c^{2}+2 b^{3} c\right\} /\left(6(p a+c)^{2}(b+p a+c)^{2}(b+c)^{2} c^{2}\right) \\
& =\frac{v_{p}(a, b, c)}{6(p a+c)^{2}(b+p a+c)^{2}(b+c)^{2} c^{2}} .
\end{aligned}
$$

Then, for $(a, b, c) \in \mathcal{P}_{p}(1)$, we have

$$
\begin{aligned}
\left(V_{p}(a, b)\right)^{2} & :=\frac{48 \pi^{2} s_{p}(a, b,(1-b-p a) / 2)^{2}}{v_{p}(a, b,(1-b-p a) / 2)} \\
& =-\frac{96 \pi^{2}\left(a^{2} p^{3}-a^{2} p^{2}-b^{2} p-p+b^{2}-1\right)^{2}}{p\left(a^{4} p^{4}-2 a^{2} b^{2} p^{2}+2 a^{2} p^{2}+b^{4}+2 b^{2}-3\right)}
\end{aligned}
$$

Note here that, in general, the function $V$ on $\mathcal{P}_{\Omega}^{G}$ is scale invariant, that is $V(d K, d a)=$ $V(K, a)$ for any $(K, a) \in \mathcal{P}_{\Omega}^{G}$ and $d>0$. Hence if $(a, b)$ is a critical point of $V_{p}(a, b)$ and $(a, b,(1-b-p a) / 2) \in \mathcal{P}_{p}(1)$, then cKEM-Futaki invariant for $(a, b,(1-b-p a) / 2)$ vanishes. The derivatives are computed as follows:

$$
\begin{aligned}
\frac{\partial V_{p}^{2}}{\partial a} & =-\frac{768 \pi^{2} a p\left(a^{2} p^{3}+b^{2} p-p-2 b^{2}+2\right)\left(a^{2} p^{3}-a^{2} p^{2}-b^{2} p-p+b^{2}-1\right)}{\left(a^{4} p^{4}-2 a^{2} b^{2} p^{2}+2 a^{2} p^{2}+b^{4}+2 b^{2}-3\right)^{2}}, \\
\frac{\partial V_{p}^{2}}{\partial b} & =\frac{768 \pi^{2} b\left(a^{2} p^{3}-a^{2} p^{2}-b^{2} p-p+b^{2}-1\right)\left(2 a^{2} p^{3}-a^{2} p^{2}-2 p-b^{2}+1\right)}{p\left(a^{4} p^{4}-2 a^{2} b^{2} p^{2}+2 a^{2} p^{2}+b^{4}+2 b^{2}-3\right)^{2}} .
\end{aligned}
$$

Both of the above vanish only when either of following holds.

$$
\begin{aligned}
& {\left[a=\frac{\sqrt{b^{2}+(p+1) /(p-1)}}{p}\right], \quad\left[a=-\frac{\sqrt{b^{2}+(p+1) /(p-1)}}{p}\right], \quad[a=0, b=0],} \\
& {[a=0, b=-\sqrt{1-2 p}], \quad[a=0, b=\sqrt{1-2 p}],} \\
& {\left[a=0, b=-\sqrt{-\frac{p}{p-1}-\frac{1}{p-1}}\right], \quad\left[a=0, b=\sqrt{-\frac{p}{p-1}-\frac{1}{p-1}}\right],}
\end{aligned}
$$




$$
\begin{aligned}
& {\left[a=-\frac{\sqrt{p-2}}{p^{3 / 2}}, b=0\right], \quad\left[a=\frac{\sqrt{p-2}}{p^{3 / 2}}, b=0\right],} \\
& {\left[a=-\frac{\sqrt{1-2 / p}}{p-1}, b=-\frac{\sqrt{1-2 p}}{p-1}\right], \quad\left[a=\frac{\sqrt{1-2 / p}}{p-1}, b=-\frac{\sqrt{1-2 p}}{p-1}\right],} \\
& {\left[a=-\frac{\sqrt{1-2 / p}}{p-1}, b=\frac{\sqrt{1-2 p}}{p-1}\right], \quad\left[a=\frac{\sqrt{1-2 / p}}{p-1}, b=\frac{\sqrt{1-2 p}}{p-1}\right] .}
\end{aligned}
$$

Case $p=1$.

$\partial V_{1}^{2} / \partial a=\partial V_{1}^{2} / \partial b=0$ if and only if $b\left(a^{2}-b^{2}-1\right)=a\left(a^{2}-b^{2}+1\right)=0$, that is $[a=0, b=0]$.

\section{Case $p>1$.}

The real solutions of $\partial V_{p}^{2} / \partial a=\partial V_{p}^{2} / \partial b=0$ are

$$
\begin{aligned}
& {\left[a=\frac{\sqrt{b^{2}+(p+1) /(p-1)}}{p}\right], \quad\left[a=-\frac{\sqrt{b^{2}+(p+1) /(p-1)}}{p}\right], \quad[a=0, b=0],} \\
& {\left[a=-\frac{\sqrt{p-2}}{p^{3 / 2}}, b=0\right], \quad\left[a=\frac{\sqrt{p-2}}{p^{3 / 2}}, b=0\right],}
\end{aligned}
$$

where the last two solutions appear only when $p>2$.

- $[a=0, b=0]$ : In this case, $(0,0,1 / 2) \in \mathcal{P}_{p}(1)$.

- $\left[a=\frac{\sqrt{b^{2}+(p+1) /(p-1)}}{p}\right]:$ In this case, we have

$$
c=\frac{1}{2}(1-b-p a)=\frac{1}{2}\left(1-b-\sqrt{b^{2}+\frac{p+1}{p-1}}\right) .
$$

But $c>0$ when $b<1 /(1-p)<0$. However we get

$$
b+c=\frac{1}{2}\left(1+b-\sqrt{b^{2}+\frac{p+1}{p-1}}\right)<0 .
$$

Hence $(a, b, c) \notin \mathcal{P}_{p}(1)$.

- $\left[a=-\frac{\sqrt{b^{2}+(p+1) /(p-1)}}{p}\right]:$ In this case we have

$$
c=\frac{1}{2}(1-b-p a)=\frac{1}{2}\left(1-b+\sqrt{b^{2}+\frac{p+1}{p-1}}\right) .
$$

$c>0$ when $b>1 /(1-p)$. However, we get

$$
p a+c=\frac{1}{2}\left(1-b-\sqrt{b^{2}+\frac{p+1}{p-1}}\right)<0 .
$$

Hence $(a, b, c) \notin \mathcal{P}_{p}(1)$. 
- $\left[a=-\frac{\sqrt{p-2}}{p^{3 / 2}}, b=0\right]:$ In this case we have

$$
\begin{aligned}
c & =\frac{1}{2}(1-b-p a)=\frac{1}{2}\left(1+\sqrt{\frac{p-2}{p}}\right)>0, \\
p a+c & =\frac{1}{2}\left(1-\sqrt{\frac{p-2}{p}}\right)>0 .
\end{aligned}
$$

Hence $(a, b, c) \in \mathcal{P}_{p}(1)$.

- $\left[a=\frac{\sqrt{p-2}}{p^{3 / 2}}, b=0\right]:$ In this case we have

$$
\begin{aligned}
c & =\frac{1}{2}(1-b-p a)=\frac{1}{2}\left(1-\sqrt{\frac{p-2}{p}}\right)>0, \\
p a+c & =\frac{1}{2}\left(1+\sqrt{\frac{p-2}{p}}\right)>0 .
\end{aligned}
$$

Hence $(a, b, c) \in \mathcal{P}_{p}(1)$.

We summarize our results of this Subsection 4.2 as follows.

Proposition 4.2. For the toric Kähler surface corresponding to $\Delta_{p}$, if $1 \leq p \leq 2$ then the cKEM-Futaki invariant vanishes only when $(a, b)=(0,0)$. If $p>2$ then the cKEM-Futaki invariant vanishes when $(a, b)=(0,0),\left( \pm \sqrt{p-2} / p^{3 / 2}, 0\right)$. In this case $p>2$, LeBrun ([21], Theorem $C$ ) shows that the Kähler class $\Omega$ contains two distinct cKEM metrics, which are ambitoric in the sense of $[\mathbf{1}]$.

Our computation complements LeBrun's result in that there are no non-Kähler solution for $1<p<2$. But this nonexistence result has been obtained by ApostolovMaschler [3] by showing the non-vanishing of the cKEM-Futaki invariant using computerassisted calculation.

\subsection{The case of the one point blow up of $\mathrm{CP}^{2}$.}

Let $\Delta_{p}$ be the convex hull of $(0,0),(p, 0),(p, 1-p),(0,1),(0<p<1)$. An affine linear function $f=a \mu_{1}+b \mu_{2}+c$ is positive on $\Delta_{p}$ if and only if

$$
c, b+c,(1-p) b+p a+c, p a+c>0 .
$$

We put

$$
\begin{aligned}
& \mathcal{P}_{p}:=\left\{(a, b, c) \in \mathbf{R}^{3} \mid c, b+c,(1-p) b+p a+c, p a+c>0\right\}, \\
& \mathcal{P}_{p}(1):=\mathcal{P}_{p} \cap\{(a, b, c) \mid(2-p) b+2 p a+4 c=1\} .
\end{aligned}
$$

For $(a, b, c) \in \mathcal{P}_{p}$, we define $s_{p}(a, b, c)$ and $v_{p}(a, b, c)$ by

$$
\int_{\partial \Delta_{p}} \frac{d \sigma}{\left(a \mu_{1}+b \mu_{2}+c\right)^{2}}
$$




$$
\begin{aligned}
= & \frac{1}{c(b+c)}+\frac{p}{(b+c)((1-p) b+p a+c)} \\
& +\frac{1-p}{((1-p) b+p a+c)(p a+c)}+\frac{p}{(p a+c) c} \\
= & \frac{\left((2 a-b) c+a^{2}-b^{2}\right) p^{2}+\left(c^{2}+2 a c+a b+b^{2}\right) p+2 c^{2}+2 b c}{c(b+c)(p a+c)((1-p) b+p a+c)} \\
= & \frac{s_{p}(a, b, c)}{c(b+c)(p a+c)((1-p) b+p a+c)} \\
\int_{\Delta_{p}} & \frac{d \mu_{1} d \mu_{2}}{\left(a \mu_{1}+b \mu_{2}+c\right)^{4}} \\
= & p\left\{-a(a-b)\left(c^{2}-2 a c+2 b c-a b+b^{2}\right) p^{3}\right. \\
& -2(2 a c-b c+a b)\left(c^{2}-2 a c+2 b c-a b+b^{2}\right) p^{2} \\
& \left.\quad+2 c(c+b)\left(3 c^{2}+3 b c+b^{2}\right)\right\} /\left(6 c^{2}(b+c)^{2}(p a+c)^{2}((1-p) b+p a+c)^{2}\right) \\
= & \frac{v_{p}(a, b, c)}{6 c^{2}(b+c)^{2}(p a+c)^{2}((1-p) b+p a+c)^{2}} .
\end{aligned}
$$

Hence for $(a, b, c) \in \mathcal{P}_{p}(1)$, we have

$$
\begin{aligned}
& V_{p}(a, b)^{2} \\
& \qquad \begin{array}{l}
:=\frac{48 \pi^{2} s_{p}(a, b,(1+(p-2) b-2 p a) / 4)^{2}}{v_{p}(a, b,(1+(p-2) b-2 p a) / 4)} \\
=96 \pi^{2}\left(3(2 a-b)^{2} p^{3}-2\left(4 a^{2}-4 a b+2 a-5 b^{2}-b\right) p^{2}-\left(20 b^{2}+1\right) p+8 b^{2}-2\right)^{2} \\
\quad / p\left((2 a-b)^{2}\left(4 a^{2}-4 a b+5 b^{2}\right) p^{5}\right. \\
\quad-2(2 a-b)\left(8 a^{3}-12 a^{2} b+8 a^{2}+6 a b^{2}-8 a b-b^{3}+6 b^{2}\right) p^{4} \\
\quad-2\left(48 a^{2} b^{2}-4 a^{2}-48 a b^{3}-16 a b^{2}+4 a b+4 b^{4}+8 b^{3}-3 b^{2}\right) p^{3} \\
\quad+4\left(16 a^{2} b^{2}-4 a^{2}-16 a b^{3}-8 a b^{2}+4 a b+2 a-12 b^{4}+4 b^{3}-5 b^{2}-b\right) p^{2} \\
\left.\quad+\left(80 b^{4}+24 b^{2}-3\right) p-32 b^{4}-16 b^{2}+6\right) .
\end{array}
\end{aligned}
$$

Then $\partial V_{p}^{2} / \partial a=\partial V_{p}^{2} / \partial b=0$ only when

(1) $a=\frac{2 \sqrt{-9 b^{2} p^{3}+\left(21 b^{2}+1\right) p^{2}+\left(1-16 b^{2}\right) p+4 b^{2}-1}+3 b p^{2}+(1-2 b) p}{6 p^{2}-4 p}$,
(2) $a=-\frac{2 \sqrt{-9 b^{2} p^{3}+\left(21 b^{2}+1\right) p^{2}+\left(1-16 b^{2}\right) p+4 b^{2}-1}-3 b p^{2}+(2 b-1) p}{6 p^{2}-4 p}$,

(3) $a=-\frac{1}{3 p^{2}-2 p} \sqrt{\frac{p^{3}-6 p^{2}+4 p}{p-2}}-\frac{1}{6 p-4} \sqrt{\frac{5 p-2}{p-2}}+\frac{1}{6 p-4}$,

$$
b=-\frac{1}{3 p-2} \sqrt{\frac{5 p-2}{p-2}},
$$


(4) $a=\frac{1}{3 p^{2}-2 p} \sqrt{\frac{p^{3}-6 p^{2}+4 p}{p-2}}-\frac{1}{6 p-4} \sqrt{\frac{5 p-2}{p-2}}+\frac{1}{6 p-4}$,

$b=-\frac{1}{3 p-2} \sqrt{\frac{5 p-2}{p-2}}$,

(5) $a=-\frac{1}{3 p^{2}-2 p} \sqrt{\frac{p^{3}-6 p^{2}+4 p}{p-2}}+\frac{1}{6 p-4} \sqrt{\frac{5 p-2}{p-2}}+\frac{1}{6 p-4}$,

$b=\frac{1}{3 p-2} \sqrt{\frac{5 p-2}{p-2}}$,

(6) $a=\frac{1}{3 p^{2}-2 p} \sqrt{\frac{p^{3}-6 p^{2}+4 p}{p-2}}+\frac{1}{6 p-4} \sqrt{\frac{5 p-2}{p-2}}+\frac{1}{6 p-4}$,

$b=\frac{1}{3 p-2} \sqrt{\frac{5 p-2}{p-2}}$,

(7) $a=\frac{p-1}{p^{2}}, \quad b=\frac{1}{p}$,

(8) $a=-\frac{1}{p^{2}}, \quad b=-\frac{1}{p}$,

(9) $a=-\frac{\sqrt{9 p^{2}-8 p}+p}{4 p^{2}}, \quad b=0$,

(10) $a=\frac{\sqrt{9 p^{2}-8 p}-p}{4 p^{2}}, \quad b=0$,

(11) $a=-\frac{1}{6 p-4} \sqrt{\frac{p^{2}+p-1}{p-1}}+\frac{1}{6 p-4}, \quad b=-\frac{1}{3 p-2} \sqrt{\frac{p^{2}+p-1}{p-1}}$,

(12) $a=\frac{1}{6 p-4} \sqrt{\frac{p^{2}+p-1}{p-1}}+\frac{1}{6 p-4}, \quad b=\frac{1}{3 p-2} \sqrt{\frac{p^{2}+p-1}{p-1}}$,

(13) $a=-\frac{\sqrt{p^{4}-4 p^{3}+16 p^{2}-16 p+4}-p^{2}+4 p-2}{2 p^{3}-4 p^{2}+12 p-8}$,

$$
b=-\frac{\sqrt{p^{4}-4 p^{3}+16 p^{2}-16 p+4}}{p^{3}-2 p^{2}+6 p-4},
$$

(14) $a=\frac{\sqrt{p^{4}-4 p^{3}+16 p^{2}-16 p+4}+p^{2}-4 p+2}{2 p^{3}-4 p^{2}+12 p-8}$,

$$
b=\frac{\sqrt{p^{4}-4 p^{3}+16 p^{2}-16 p+4}}{p^{3}-2 p^{2}+6 p-4},
$$

(15) $a=-\frac{-p+2 \sqrt{1-p}+2}{2 p^{2}}, \quad b=0$, 
(16) $a=\frac{p+2 \sqrt{1-p}-2}{2 p^{2}}, \quad b=0$.

Up to this point in this subsection, we used Maxima to derive the above conclusions. Among the above, real solutions are the following:

(1), (2), (7), (8), (15), (16),

(9), (10): when $1>p \geq 8 / 9$

(11), (12): when $0<p<(\sqrt{5}-1) / 2$

(13), (14) : when $0<p \leq \alpha, \beta \leq p<1$, where $0<\alpha<\beta<1$ are the real roots of $p^{4}-4 p^{3}+16 p^{2}-16 p+4=0$.

Let us check the cases $(7)-(16)$ whether $(a, b, c) \in \mathcal{P}_{p}(1)$ or not. The proof of each case is elementary. We only give a detailed proof only for (11) and (13) for the reader's convenience. We leave the cases (1) and (2) to later study.

(7) : $(a, b, c) \notin \mathcal{P}_{p}(1)$ since $c=(1+(p-2) b-2 p a) / 4=0$.

(8) : $(a, b, c) \notin \mathcal{P}_{p}(1)$ since $b+c=0$.

(9) : $(a, b, c) \in \mathcal{P}_{p}(1)$ since

$$
c=b+c=\frac{3}{8}+\frac{\sqrt{9 p^{2}-8 p}}{8 p}>0
$$

and

$$
(1-p) b+p a+c=p a+c=\frac{1}{8}-\frac{\sqrt{9 p^{2}-8 p}}{8 p}>0 .
$$

(10) $:(a, b, c) \in \mathcal{P}_{p}(1)$ since

$$
c=b+c=\frac{3}{8}-\frac{\sqrt{9 p^{2}-8 p}}{8 p}>0
$$

and

$$
(1-p) b+p a+c=p a+c=\frac{1}{8}+\frac{\sqrt{9 p^{2}-8 p}}{8 p}>0 .
$$

(11) : $(a, b, c) \notin \mathcal{P}_{p}(1)$

Proof. First of all, recall $0<p<(\sqrt{5}-1) / 2$. We need to check the signs of

$$
\begin{aligned}
c & =\frac{\sqrt{\left(p^{2}+p-1\right) /(p-1)}+p-1}{6 p-4}, \\
b+c & =-\frac{\sqrt{\left(p^{2}+p-1\right) /(p-1)}-p+1}{6 p-4}, \\
(1-p) b+p a+c & =\frac{(p-1) \sqrt{\left(p^{2}+p-1\right) /(p-1)}+2 p-1}{6 p-4}
\end{aligned}
$$


and

$$
p a+c=-\frac{(p-1) \sqrt{\left(p^{2}+p-1\right) /(p-1)}-2 p+1}{6 p-4} .
$$

Since $4-6 p>0$, it is sufficient to prove that

$$
(4-6 p)(p a+c)=(p-1) \sqrt{\frac{p^{2}+p-1}{p-1}}-2 p+1<0 .
$$

When $1 / 2 \leq p<(\sqrt{5}-1) / 2,(33)$ is trivial. When $0<p<1 / 2$,

$$
\begin{aligned}
(p-1) \sqrt{\frac{p^{2}+p-1}{p-1}}<2 p-1 & \Longleftrightarrow \sqrt{\frac{p^{2}+p-1}{p-1}}>\frac{2 p-1}{p-1} \\
& \Longleftrightarrow \frac{p^{2}+p-1}{p-1}>\frac{(2 p-1)^{2}}{(p-1)^{2}} \\
& \Longleftrightarrow p^{2}-4 p+2>0 .
\end{aligned}
$$

This completes the proof.

(12) : $(a, b, c) \notin \mathcal{P}_{p}(1)$ since

$$
\begin{aligned}
c & =-\frac{\sqrt{\left(p^{2}+p-1\right) /(p-1)}-p+1}{6 p-4}, \\
b+c & =\frac{\sqrt{\left(p^{2}+p-1\right) /(p-1)}+p-1}{6 p-4}, \\
(1-p) b+p a+c & =-\frac{(p-1) \sqrt{\left(p^{2}+p-1\right) /(p-1)}-2 p+1}{6 p-4}<0
\end{aligned}
$$

and

$$
p a+c=\frac{(p-1) \sqrt{\left(p^{2}+p-1\right) /(p-1)}+2 p-1}{6 p-4} .
$$

(13) : If $0<p \leq \alpha$ then $(a, b, c) \in \mathcal{P}_{p}(1)$. If $\beta \leq p<1$, then $(a, b, c) \notin \mathcal{P}_{p}(1)$. This is because

$$
\begin{aligned}
c & =\frac{\sqrt{p^{4}-4 p^{3}+16 p^{2}-16 p+4}+p^{2}+2 p-2}{2\left(p^{3}-2 p^{2}+6 p-4\right)}, \\
b+c & =-\frac{\sqrt{p^{4}-4 p^{3}+16 p^{2}-16 p+4}-p^{2}-2 p+2}{2\left(p^{3}-2 p^{2}+6 p-4\right)} \\
(1-p) b+p a+c & =\frac{(p-1)\left(\sqrt{p^{4}-4 p^{3}+16 p^{2}-16 p+4}+p^{2}-2 p+2\right)}{2\left(p^{3}-2 p^{2}+6 p-4\right)} \\
p a+c & =-\frac{(p-1)\left(\sqrt{p^{4}-4 p^{3}+16 p^{2}-16 p+4}-p^{2}+2 p-2\right)}{2\left(p^{3}-2 p^{2}+6 p-4\right)} .
\end{aligned}
$$


When $0<p \leq \alpha$, then $c, b+c,(1-p) b+p a+c$ and $p a+c$ are positive. On the other hand, when $\beta \leq p<1$, we have $(1-p) b+p a+c<0$.

Proof. For $0<p \leq \alpha=0.386 \cdots,(a, b, c) \in \mathcal{P}_{p}(1)$

$$
\begin{aligned}
c & =\frac{\sqrt{p^{4}-4 p^{3}+16 p^{2}-16 p+4}+p^{2}+2 p-2}{2\left(p^{3}-2 p^{2}+6 p-4\right)}, \\
b+c & =\frac{-\sqrt{p^{4}-4 p^{3}+16 p^{2}-16 p+4}+p^{2}+2 p-2}{2\left(p^{3}-2 p^{2}+6 p-4\right)} \\
(1-p) b+p a+c & =(1-p) \frac{-\sqrt{p^{4}-4 p^{3}+16 p^{2}-16 p+4}-p^{2}+2 p-2}{2\left(p^{3}-2 p^{2}+6 p-4\right)}, \\
p a+c & =(1-p) \frac{\sqrt{p^{4}-4 p^{3}+16 p^{2}-16 p+4}-p^{2}+2 p-2}{2\left(p^{3}-2 p^{2}+6 p-4\right)} .
\end{aligned}
$$

It is easy to see that $p^{3}-2 p^{2}+6 p-4$ is negative on $(0, \alpha]$. So, to prove $c, b+c,(1-$ $p) b+p a+c, p a+c>0$, it is sufficient to see that

$$
\begin{aligned}
A & :=2\left(p^{3}-2 p^{2}+6 p-4\right) c \\
& =\sqrt{p^{4}-4 p^{3}+16 p^{2}-16 p+4}+p^{2}+2 p-2<0, \\
B & :=2\left(p^{3}-2 p^{2}+6 p-4\right)(b+c) \\
& =-\sqrt{p^{4}-4 p^{3}+16 p^{2}-16 p+4}+p^{2}+2 p-2<0, \\
C & :=\frac{2\left(p^{3}-2 p^{2}+6 p-4\right)((1-p) b+p a+c)}{1-p} \\
& =-\sqrt{p^{4}-4 p^{3}+16 p^{2}-16 p+4}-p^{2}+2 p-2<0, \\
D & :=\frac{2\left(p^{3}-2 p^{2}+6 p-4\right)(p a+c)}{1-p} \\
& =\sqrt{p^{4}-4 p^{3}+16 p^{2}-16 p+4}-p^{2}+2 p-2<0 .
\end{aligned}
$$

Since $A \geq B$ and $A \geq D \geq C$, it sufficient to see $A<0$. For $0<p \leq \alpha$, $p^{4}-4 p^{3}+16 p^{2}-16 p+4,-p^{2}-2 p+2>0$. Therefore

$$
A<0 \Longleftrightarrow p^{4}-4 p^{3}+16 p^{2}-16 p+4<\left(-p^{2}-2 p+2\right)^{2} \Longleftrightarrow-8(p-1)^{2} p<0 .
$$

Thus we are done for $0<p \leq \alpha$.

For $\beta=0.844 \cdots \leq p<1$, it is easy to see that $p^{3}-2 p^{2}+6 p-4$ and $p^{2}-2 p+2$ are positive. Hence

$$
(1-p) b+p a+c=\frac{(p-1)\left(\sqrt{p^{4}-4 p^{3}+16 p^{2}-16 p+4}+p^{2}-2 p+2\right)}{2\left(p^{3}-2 p^{2}+6 p-4\right)}<0 .
$$

Thus we are done for $\beta \leq p<1$. 
(14) : If $0<p \leq \alpha$, then $(a, b, c) \in \mathcal{P}_{p}(1)$. If $\beta \leq p<1$, then $(a, b, c) \notin \mathcal{P}_{p}(1)$.

$$
\begin{aligned}
c & =-\frac{\sqrt{p^{4}-4 p^{3}+16 p^{2}-16 p+4}-p^{2}-2 p+2}{2\left(p^{3}-2 p^{2}+6 p-4\right)}, \\
b+c & =\frac{\sqrt{p^{4}-4 p^{3}+16 p^{2}-16 p+4}+p^{2}+2 p-2}{2\left(p^{3}-2 p^{2}+6 p-4\right)}, \\
(1-p) b+p a+c & =-\frac{(p-1)\left(\sqrt{p^{4}-4 p^{3}+16 p^{2}-16 p+4}-p^{2}+2 p-2\right)}{2\left(p^{3}-2 p^{2}+6 p-4\right)}, \\
p a+c & =\frac{(p-1)\left(\sqrt{p^{4}-4 p^{3}+16 p^{2}-16 p+4}+p^{2}-2 p+2\right)}{2\left(p^{3}-2 p^{2}+6 p-4\right)} .
\end{aligned}
$$

When $0<p \leq \alpha \beta \leq p<1, c, b+c,(1-p) b+p a+c$ and $p a+c$ are positive. On the other hand, when $\beta \leq p<1$, we have $(1-p) b+p a+c<0$.

(15) : $(a, b, c) \notin \mathcal{P}_{p}(1)$

$$
\begin{gathered}
c=b+c=\frac{\sqrt{1-p}+1}{2 p}, \\
p a+c=(1-p) b+p a+c=-\frac{-p+\sqrt{1-p}+1}{2 p}<0 .
\end{gathered}
$$

(16) : $(a, b, c) \in \mathcal{P}_{p}(1)$

$$
\begin{gathered}
c=b+c=-\frac{\sqrt{1-p}-1}{2 p}>0 \\
(1-p) b+p a+c=p a+c=\frac{p+\sqrt{1-p}-1}{2 p}>0 .
\end{gathered}
$$

We record the data of the cases (1), (2) for later study.

(1):

$$
\begin{aligned}
c=- & \frac{\sqrt{-9 b^{2} p^{3}+\left(21 b^{2}+1\right) p^{2}+\left(1-16 b^{2}\right) p+4 b^{2}-1}+(3 b-1) p-2 b+1}{6 p-4}, \\
b+c & = \\
& \quad-\frac{\sqrt{-9 b^{2} p^{3}+\left(21 b^{2}+1\right) p^{2}+\left(1-16 b^{2}\right) p+4 b^{2}-1}+(-3 b-1) p+2 b+1}{6 p-4}, \\
(1-p) b+p a+c= & \frac{\sqrt{-9 b^{2} p^{3}+\left(21 b^{2}+1\right) p^{2}+\left(1-16 b^{2}\right) p+4 b^{2}-1}+(3 b+3) p-2 b-1}{6 p-4}, \\
p a+ & c= \\
& \quad \frac{\sqrt{-9 b^{2} p^{3}+\left(21 b^{2}+1\right) p^{2}+\left(1-16 b^{2}\right) p+4 b^{2}-1}+6 b p^{2}+(3-7 b) p+2 b-1}{6 p-4} .
\end{aligned}
$$


(2):

$$
\begin{aligned}
c= & \frac{\sqrt{-9 b^{2} p^{3}+\left(21 b^{2}+1\right) p^{2}+\left(1-16 b^{2}\right) p+4 b^{2}-1}+(1-3 b) p+2 b-1}{6 p-4}, \\
b+ & c= \\
& \frac{\sqrt{-9 b^{2} p^{3}+\left(21 b^{2}+1\right) p^{2}+\left(1-16 b^{2}\right) p+4 b^{2}-1}+(3 b+1) p-2 b-1}{6 p-4}, \\
& (1-p) b+p a+c= \\
& -\frac{\sqrt{-9 b^{2} p^{3}+\left(21 b^{2}+1\right) p^{2}+\left(1-16 b^{2}\right) p+4 b^{2}-1}+3 b p^{2}+(-5 b-2) p+2 b+1}{6 p-4}, \\
p a & +c= \\
& -\frac{\sqrt{-9 b^{2} p^{3}+\left(21 b^{2}+1\right) p^{2}+\left(1-16 b^{2}\right) p+4 b^{2}-1}-3 b p^{2}+(5 b-2) p-2 b+1}{6 p-4} .
\end{aligned}
$$

To sum up, leaving (1), (2) aside, if $0<p<\alpha$ then the cKEM-Futaki invariant vanishes for (13), (14), and (16). If $\alpha \leq p \leq 8 / 9$ then the cKEM-Futaki invariant vanishes only for (16). If $8 / 9<p<1$ then cKEM-Futaki invariant vanishes for (9), (10) and (16). We wish to compare this with the following result of LeBrun.

TheOrem 4.3 (LeBrun [22]). Let $M$ be the blow-up of $\mathbf{C P}^{2}$ at one point.

(a) For any Kähler class, there exists a Kähler metric which is conformal to a conformally Kähler, Einstein-Maxwell metric.

(b) Express an arbitrary Kähler class as $\Omega=u \mathcal{L}-v \mathcal{E}$ where $\mathcal{L}$ and $\mathcal{E}$ are the Poincaré duals of a projective line and the exceptional divisor. If $9<u / v$ then there are two Kähler metrics which are conformal to a conformally Kähler, Einstein-Maxwell metric. One of these metrics $g$ has two positive potential functions $f$ of Hamiltonian Killing vector fields such that $\tilde{g}=f^{-2} g$ is a conformally Kähler, EinsteinMaxwell metric. Further there is an orientation reversing isometry between these two conformally Kähler, Einstein-Maxwell metrics $\tilde{g}$.

All the Kähler metrics and positive potential functions of Hamiltonian Killing vector fields in (a) and (b) are $U(2)$-invariant, and there are no other $U(2)$-invariant conformally Kähler, Einstein-Maxwell metrics.

THEOREM 4.4. Let $M$ be the blow-up of $\mathbf{C P}^{2}$ at one point, $\Delta_{p}$ the convex hull of $(0,0),(p, 0),(p, 1-p),(0,1),(0<p<1)$ in $\left(\mu_{1}, \mu_{2}\right)$-plane, and consider $\Delta_{p}$ as the moment map image of $M$. Let $0<\alpha<\beta<1$ be the real roots of

$$
p^{4}-4 p^{3}+16 p^{2}-16 p+4=0 .
$$

(a) For $0<p<1$, the affine function

$$
f=\frac{p+2 \sqrt{1-p}-2}{2 p^{2}} \mu_{1}-\frac{\sqrt{1-p}-1}{2 p}
$$


corresponds to the conformally Kähler, Einstein-Maxwell metric in (a) in Theorem 4.3.

(b) For $8 / 9<p<1$, the two affine functions

$$
\begin{aligned}
& f=-\frac{\sqrt{9 p^{2}-8 p}+p}{4 p^{2}} \mu_{1}+\frac{3}{8}+\frac{\sqrt{9 p^{2}-8 p}}{8 p}, \\
& f=\frac{\sqrt{9 p^{2}-8 p}-p}{4 p^{2}} \mu_{1}+\frac{3}{8}-\frac{\sqrt{9 p^{2}-8 p}}{8 p}
\end{aligned}
$$

correspond to the conformally Kähler, Einstein-Maxwell metric in (b) in Theorem 4.3 .

(c) For $0<p<\alpha$, the two affine functions

$$
\begin{aligned}
f= & -\frac{\sqrt{p^{4}-4 p^{3}+16 p^{2}-16 p+4}-p^{2}+4 p-2}{2 p^{3}-4 p^{2}+12 p-8} \mu_{1} \\
& -\frac{\sqrt{p^{4}-4 p^{3}+16 p^{2}-16 p+4}}{p^{3}-2 p^{2}+6 p-4} \mu_{2} \\
& +\frac{\sqrt{p^{4}-4 p^{3}+16 p^{2}-16 p+4}+p^{2}+2 p-2}{2\left(p^{3}-2 p^{2}+6 p-4\right)} \\
f= & \frac{\sqrt{p^{4}-4 p^{3}+16 p^{2}-16 p+4}+p^{2}-4 p+2}{2 p^{3}-4 p^{2}+12 p-8} \mu_{1} \\
& +\frac{\sqrt{p^{4}-4 p^{3}+16 p^{2}-16 p+4}}{p^{3}-2 p^{2}+6 p-4} \mu_{2} \\
& -\frac{\sqrt{p^{4}-4 p^{3}+16 p^{2}-16 p+4}-p^{2}-2 p+2}{2\left(p^{3}-2 p^{2}+6 p-4\right)}
\end{aligned}
$$

are positive and satisfy $\mathrm{Fut}_{f}=0$. If there is a Kähler metric $g$ such that $f^{-2} g$ gives a conformally Kähler, Einstein-Maxwell metric then it has $U(1) \times U(1)$-symmetry.

Proof. Recall that, in the classification (1)-(16), the cases with $F u t_{f}=0$ are the cases (1), (2), (9), (10), (13), (14) and (16). A toric cKEM metric in these cases, if any, has $U(2)$-symmetry if and only if $b=0$.

We see that (1) with $b=0$ or (2) with $b=0$ do not occur. In fact, if (1) with $b=0$ occurs then

$$
a=\frac{2 \sqrt{p^{2}+p-1}+p}{6 p^{2}-4 p}, \quad 4 c=\frac{2 p-2-\sqrt{2 p^{2}+p-1}}{3 p-2} .
$$

We have to have $p^{2}+p-1>0$, and thus we have only to consider the case $(\sqrt{5}-1) / 2<$ $p<1$. In this range, we have

$$
\frac{p a+c}{c}=\frac{1+2 p a}{1-2 p a}=\frac{-\sqrt{p^{2}+p-1}-2 p+1}{\sqrt{p^{2}+p-1}+1-p}<0 .
$$


Hence $c$ or $p a+c$ is negative. So $f(0,0)<0$ or $f(p, 0)<0$. If $(2)$ with $b=0$ occurs then

$$
a=-\frac{2 \sqrt{p^{2}+p-1}-p}{6 p^{2}-4 p}, \quad p a+c=\frac{-\sqrt{p^{2}+p-1}+2 p-1}{6 p-4} .
$$

For $(\sqrt{5}-1) / 2<p<1$ we have $p a+c<0$ since the numerator and denominator both change sign at $p=2 / 3$ and $p a+c=-3 / 4$ at $p=2 / 3$. So $f(p, 0)<0$.

It follows that $U(2)$-symmetry occurs exactly when $(9),(10),(16)$ because we have $b=0$ in the cases (9), (10) and (16).

The moment map image $\Delta_{p}$ determines the Kähler class $\Omega=u \mathcal{L}-v \mathcal{E}$ with $u=1$ and $v=1-p$. Thus $u / v \leq 9$ if and only if $p \leq 8 / 9$. In this region, only the case (16) allows an $f$ with vanishing cKEM-Futaki invariant, and in fact Theorem 4.3 shows there is one cKEM metric with $U(2)$-symmetry. Moreover by Theorem 3 in [3], for a given $f$, a toric Kähler metric $g$ such that $f^{-2} g$ is a cKEM metric is unique. Thus (a) holds.

In the region $u / v>9$, that is, $p>8 / 9$, the case (9), (10) and (16) gives an $f$ with vanishing cKEM-Futaki invariant. By the similar arguments as in the case of $p \leq 8 / 9$, these three cases correspond to the three LeBrun solutions in Theorem 4.3 cited above. Moreover the cases (9) and (10) correspond to (b) in Theorem 4.3, which can be checked by computing $V_{p}(a, 0)^{2}$ in (32). Put

$$
\begin{aligned}
f(a, p) & :=\frac{V_{p}(a, 0)^{2}}{96 \pi^{2}} \\
& =\frac{\left(12 a^{2} p^{3}-4 a(2 a+1) p^{2}-p-2\right)^{2}}{p\left(16 a^{4} p^{5}-32 a^{3}(a+1) p^{4}+8 a^{2} p^{3}+8 a(1-2 a) p^{2}-3 p+6\right)} .
\end{aligned}
$$

Then we have

$$
f\left(-\frac{\sqrt{9 p^{2}-8 p}+p}{4 p^{2}}, p\right)=f\left(\frac{\sqrt{9 p^{2}-8 p}-p}{4 p^{2}}, p\right)=5-\frac{2}{p},
$$

which shows the solutions corresponding to (9) and (10) are homothetic. (These are not isometric since the total scalar curvature and the volume have different values.) But for the case (16) the value of $f(a, p)$ is not equal to the cases (9) and (10) because

$$
\begin{aligned}
f & \left(\frac{p+2 \sqrt{1-p}-2}{2 p^{2}}, p\right) \\
& =-\frac{4 p^{4}+\sqrt{1-p}\left(24 p^{3}-112 p^{2}+112 p-32\right)-68 p^{3}+164 p^{2}-128 p+32}{p^{4}+6 p^{3}+\sqrt{1-p}\left(16 p^{2}-16 p\right)-24 p^{2}+16 p}
\end{aligned}
$$

and

$$
\begin{gathered}
f\left(-\frac{\sqrt{9 p^{2}-8 p}+p}{4 p^{2}}, p\right)-f\left(\frac{p+2 \sqrt{1-p}-2}{2 p^{2}}, p\right) \\
=\frac{9 p^{3}+\sqrt{1-p}\left(24 p^{2}-32 p\right)-40 p^{2}+32 p}{p^{3}+6 p^{2}+\sqrt{1-p}(16 p-16)-24 p+16} .
\end{gathered}
$$

Hence we have proved (b). 
The statement (c) is the possibility of the cases (13) and (14). This completes the proof of Theorem 4.4.

We have not been able to construct a cKEM metric for the cases (13) and (14). There is an ansatz to construct local and global ambitoric solutions, see $[\mathbf{1}],[\mathbf{2}],[\mathbf{3}]$. We have not been able to rule out the cases (1) and (2). We leave these problems to the interested readers.

\subsection{Hirzebruch surfaces.}

Let $\Delta_{p, q}$ be the convex hull of $(0,0),(p, 0),(p,(1-p) q),(0, q),(0<p<1, q \in$ $\mathbf{N})$. An affine linear function $f=a \mu_{1}+b \mu_{2}+c$ is positive on $\Delta_{p, q}$ if and only if $c, q b+c,(1-p) q b+p a+c, p a+c>0$. We put

$$
\begin{aligned}
& \mathcal{P}_{p, q}:=\left\{(a, b, c) \in \mathbf{R}^{3} \mid c, q b+c,(1-p) q b+p a+c, p a+c>0\right\}, \\
& \mathcal{P}_{p, q}(1):=\mathcal{P}_{p} \cap\{(a, b, c) \mid(2-p) q b+2 p a+4 c=1\} .
\end{aligned}
$$

For $(a, b, c) \in \mathcal{P}_{p, q}$, we have

$$
\begin{aligned}
& \int_{\partial \Delta_{p, q}} \frac{d \sigma}{\left(a \mu_{1}+b \mu_{2}+c\right)^{2}} \\
& =\frac{q}{c(q b+c)}+\frac{p}{(q b+c)((1-p) q b+p a+c)} \\
& +\frac{(1-p) q}{(p a+c)((1-p) q b+p a+c)}+\frac{p}{c(p a+c)} \\
& =-\left\{\left(\left(a b+b^{2}\right) q^{2}+\left(b c-a^{2}-a b\right) q-2 a c\right) p^{2}\right. \\
& \left.+\left(\left(2 b c-a b-b^{2}\right) q^{2}+\left(c^{2}-2(a+b) c\right) q-2 c^{2}\right) p-2 c q(q b+c)\right\} \\
& /\{(p a+c)((1-p) q b+p a+c)(q b+c) c\} \\
& =\frac{s_{p, q}(a, b, c)}{(p a+c)((1-p) q b+p a+c)(q b+c) c}, \\
& \int_{\Delta_{p}} \frac{d \mu_{1} d \mu_{2}}{\left(a \mu_{1}+b \mu_{2}+c\right)^{4}} \\
& =\left\{p q \left(a b^{3} p^{3} q^{3}+2 b^{3} c p^{2} q^{3}-2 a b^{3} p^{2} q^{3}-4 b^{3} c p q^{3}+a b^{3} p q^{3}+2 b^{3} c q^{3}\right.\right. \\
& +2 a b^{2} c p^{3} q^{2}-2 a^{2} b^{2} p^{3} q^{2}+4 b^{2} c^{2} p^{2} q^{2}-10 a b^{2} c p^{2} q^{2}+2 a^{2} b^{2} p^{2} q^{2} \\
& -12 b^{2} c^{2} p q^{2}+8 a b^{2} c p q^{2}+8 b^{2} c^{2} q^{2}+a b c^{2} p^{3} q-4 a^{2} b c p^{3} q+a^{3} b p^{3} q \\
& +2 b c^{3} p^{2} q-14 a b c^{2} p^{2} q+8 a^{2} b c p^{2} q-12 b c^{3} p q+18 a b c^{2} p q+12 b c^{3} q \\
& \left.\left.-a^{2} c^{2} p^{3}+2 a^{3} c p^{3}-4 a c^{3} p^{2}+8 a^{2} c^{2} p^{2}-3 c^{4} p+12 a c^{3} p+6 c^{4}\right)\right\} \\
& /\left\{6 c^{2}(p a+c)^{2}(q b+c)^{2}((1-p) q b+p a+c)^{2}\right\} \\
& =\frac{v_{p, q}(a, b, c)}{6 c^{2}(p a+c)^{2}(q b+c)^{2}((1-p) q b+p a+c)^{2}} \text {. }
\end{aligned}
$$

Hence for $(a, b, c) \in \mathcal{P}_{p, q}(1)$, we have 


$$
\begin{aligned}
& V_{p, q}(a, b)^{2} \\
& :=\frac{48 \pi^{2} s_{p, q}(a, b,(1+(p-2) q b-2 p a) / 4)^{2}}{v_{p, q}(a, b,(1+(p-2) q b-2 p a) / 4)} \\
& =\left\{6 \left(b^{2} p^{3} q^{3}+2 b^{2} p^{2} q^{3}-12 b^{2} p q^{3}+8 b^{2} q^{3}-4 a b p^{3} q^{2}+2 b^{2} p^{3} q^{2}\right.\right. \\
& +8 a b p^{2} q^{2}+8 b^{2} p^{2} q^{2}+2 b p^{2} q^{2}-8 b^{2} p q^{2}+4 a^{2} p^{3} q-8 a b p^{3} q \\
& \left.\left.-8 a^{2} p^{2} q-4 a p^{2} q+p q-2 q+8 a^{2} p^{3}-2 p\right)^{2}\right\} \\
& /\left\{p q \left(5 b^{4} p^{5} q^{4}-2 b^{4} p^{4} q^{4}-8 b^{4} p^{3} q^{4}-48 b^{4} p^{2} q^{4}+80 b^{4} p q^{4}-32 b^{4} q^{4}\right.\right. \\
& -24 a b^{3} p^{5} q^{3}+16 a b^{3} p^{4} q^{3}+12 b^{3} p^{4} q^{3}+96 a b^{3} p^{3} q^{3}-16 b^{3} p^{3} q^{3}-64 a b^{3} p^{2} q^{3} \\
& +16 b^{3} p^{2} q^{3}+40 a^{2} b^{2} p^{5} q^{2}-48 a^{2} b^{2} p^{4} q^{2}-40 a b^{2} p^{4} q^{2}-96 a^{2} b^{2} p^{3} q^{2}+32 a b^{2} p^{3} q^{2} \\
& +6 b^{2} p^{3} q^{2}+64 a^{2} b^{2} p^{2} q^{2}-32 a b^{2} p^{2} q^{2}-20 b^{2} p^{2} q^{2}+24 b^{2} p q^{2}-16 b^{2} q^{2} \\
& -32 a^{3} b p^{5} q+64 a^{3} b p^{4} q+48 a^{2} b p^{4} q-8 a b p^{3} q+16 a b p^{2} q-4 b p^{2} q+16 a^{4} p^{5} \\
& \left.\left.-32 a^{4} p^{4}-32 a^{3} p^{4}+8 a^{2} p^{3}-16 a^{2} p^{2}+8 a p^{2}-3 p+6\right)\right\} \text {. }
\end{aligned}
$$

For example, the following are real solutions of $\partial V_{p, q}^{2} / \partial a=\partial V_{p, q}^{2} / \partial b=0$ :

$$
\begin{aligned}
& \text { (1) }\left[a=\frac{p+2 \sqrt{1-p}-2}{2 p^{2}}, b=0\right], \\
& \text { (2) }\left[a=\frac{ \pm \sqrt{p\left(p q^{2}+4 q(p-2)-4 p\right)}-p q}{4 p^{2}}, b=0\right], \\
& \text { (3) }\left[a=-\frac{\sqrt{4(1-p)^{2} q^{2}-4(p-1)(p-2) p q+p^{4}}-2(p-1) q+p(p-2)}{2\left(2(p-1)(p-2) q-p^{3}\right)},\right. \\
& \left.\quad b=-\frac{\sqrt{4(1-p)^{2} q^{2}-4(p-1)(p-2) p q+p^{4}}}{q\left(2(p-1)(p-2) q-p^{3}\right)}\right], \\
& \text { (4) }\left[a=\frac{\sqrt{4(1-p)^{2} q^{2}-4(p-1)(p-2) p q+p^{4}}+2(p-1) q-p(p-2)}{2\left(2(p-1)(p-2) q-p^{3}\right)},\right. \\
& \left.b=\frac{\sqrt{4(1-p)^{2} q^{2}-4(p-1)(p-2) p q+p^{4}}}{q\left(2(p-1)(p-2) q-p^{3}\right)}\right] .
\end{aligned}
$$

Let us check these four cases.

(1) $\left[a=\frac{p+2 \sqrt{1-p}-2}{2 p^{2}}, b=0\right]:(a, b, c) \in \mathcal{P}_{p, q}(1)$.

In this case, $c=q b+c, p a+c=(1-p) q b+p a+c$ are independent of $q$. Hence these are positive by the computation when $q=1$.

(2) $\left[a=\frac{ \pm \sqrt{p\left(p q^{2}+4 q(p-2)-4 p\right)}-p q}{4 p^{2}}, b=0\right]:(a, b, c) \notin \mathcal{P}_{p, q}(1)$.

When $q=2$, these are not real solutions. When $q \geq 3$ and

$$
p q^{2}+4 q(p-2)-4 p>0,
$$


we have

(3)

$$
\begin{aligned}
{[a} & =-\frac{\sqrt{4(1-p)^{2} q^{2}-4(p-1)(p-2) p q+p^{4}}-2(p-1) q+p(p-2)}{2\left(2(p-1)(p-2) q-p^{3}\right)} \\
b & \left.=-\frac{\sqrt{4(1-p)^{2} q^{2}-4(p-1)(p-2) p q+p^{4}}}{q\left(2(p-1)(p-2) q-p^{3}\right)}\right]
\end{aligned}
$$

(4) $\left[a=\frac{\sqrt{4(1-p)^{2} q^{2}-4(p-1)(p-2) p q+p^{4}}+2(p-1) q-p(p-2)}{2\left(2(p-1)(p-2) q-p^{3}\right)}\right.$,

$$
\left.b=\frac{\sqrt{4(1-p)^{2} q^{2}-4(p-1)(p-2) p q+p^{4}}}{q\left(2(p-1)(p-2) q-p^{3}\right)}\right] .
$$

Take $q=2,3,4$ and perform a numerical analysis, then we see that there are two roots $0<\alpha_{q}<\beta_{q}<1$ of the quartic equation in $p$ :

$$
4(1-p)^{2} q^{2}-4(p-1)(p-2) p q+p^{4}=0,
$$

and that for $0<p<\alpha_{q}$, we have $(a, b, c) \in \mathcal{P}_{p, q}(1)$ so that the cKEM-Futaki invariant vanishes.

We conclude this section with the following two remarks.

REMARK 4.5. It is likely that the case (1) corresponds to LeBrun's construction in [22], Theorem $\mathrm{D}$ with $k \geq 2$. This case should be the only case with $U(2)$-symmetry. We may prove it by showing $b=0$ occurs only in the case (1).

REMARK 4.6. It would be interesting if one can prove or disprove the existence of cKEM metrics in the cases of (3) and (4) with $0<p<\alpha_{q}$ since, if any, the solutions necessarily have $U(1) \times U(1)$-symmetry.

\section{References}

[1] V. Apostolov, D. M. J. Calderbank and P. Gauduchon, Ambitoric geometry I: Einstein metrics and extremal ambikähler structures, J. Reine Angew. Math., 721 (2016), 109-147.

[2] V. Apostolov, D. M. J. Calderbank and P. Gauduchon, Ambitoric geometry II: extremal toric surfaces and Einstein 4-orbifolds, Ann. Sci. Éc. Norm. Supér. (4), 48 (2015), 1075-1112.

[ 3 ] V. Apostolov and G. Maschler, Conformally Kähler, Einstein-Maxwell geometry, to appear in J. Eur. Math. Soc. (JEMS).

[4] L. Bérard-Bergery, Sur de nouvelles variétés riemanniennes d'Einstein, (French) [Some new Einstein Riemannian manifolds] Institut Élie Cartan, 6, 1-60, Inst. Élie Cartan, 6, Univ. Nancy, Nancy, 1982.

[ 5 ] A. Besse, Einstein manifolds, Springer-Verlag, Berlin-Heidelberg-Tokyo, 1987.

[6] C. P. Boyer, K. Galicki and S. R. Simanca Canonical Sasakian metrics, Comm. Math. Phys., 279 (2008), 705-733.

[ 7 ] X.-X. Chen, C. LeBrun and B. Weber, On conformally Kähler, Einstein manifolds, J. Amer. Math. Soc., 21 (2008), 1137-1168.

[ 8 ] K. Cho, A. Futaki and H. Ono, Uniqueness and examples of toric Sasaki-Einstein metrics, Comm. Math. Phys., 277 (2008), 439-458. 
[ 9 ] S. K. Donaldson, Remarks on gauge theory, complex geometry and 4-manifold topology: In 'Fields Medallists Lectures', World Sci. Ser. 20th Century Math., 5, World Sci. Publ., River Edeg, NJ, 1997, 384-403.

[10] S. K. Donaldson, Scalar curvature and stability of toric varieties, J. Differential Geometry, 62 (2002), 289-349.

[11] S. K. Donaldson, Constant scalar curvature metrics on toric surfaces, Geom. Funct. Anal., 19 (2009), 83-136.

[12] A. Fujiki, Moduli space of polarized algebraic manifolds and Kähler metrics, Sugaku Expositions, 5 (1992), 173-191.

[13] A. Futaki, An obstruction to the existence of Einstein Kähler metrics, Invent. Math., 73 (1983), $437-443$.

[14] A. Futaki, On compact Kähler manifolds of constant scalar curvature, Proc. Japan Acad., Ser. A, 59 (1983), 401-402.

[15] A. Futaki and H. Ono, Einstein metrics and GIT stability, Sugaku Expositions, 24 (2011), 93-122. (Translated from Sugaku, 60 (2008), 175-202 in Japanese.)

[16] A. Futaki and H. Ono, Conformally Einstein-Maxwell Kähler metrics and the structure of the automorphisms, to appear in Math. Zeit.

[17] A. Futaki, H. Ono and G. Wang, Transverse Kähler geometry of Sasaki manifolds and toric SasakiEinstein manifolds, J. Differential Geom., 83 (2009), 585-635.

[18] C. Koca and C. W. Tønnesen-Friedman, Strongly Hermitian Einstein-Maxwell solutions on ruled surfaces, Ann. Glob. Annl. Geom., 50 (2016), 29-46.

[19] A. Lahdili, Automorphisms and deformations of conformally Kähler, Einstein-Maxwell metrics, J. Geom. Anal. (2018) online.

[20] C. LeBrun, On Einstein, Hermitian 4-manifolds, J. Differential Geom., 90 (2012), 277-302.

[21] C. LeBrun, The Einstein-Maxwell equations, Kähler metrics, and Hermitian geometry, J. Geom. Phys., 91 (2015), 163-171.

[22] C. LeBrun, The Einstein-Maxwell equations and conformally Kähler geometry, Commun. Math. Phys., 344 (2016), 621-653.

[23] E. Legendre, Toric geometry of convex quadrilaterals, J. Symplectic Geom., 9 (2011), 343-385.

[24] D. Martelli, J. Sparks and S.-T. Yau, Sasaki-Einstein manifolds and volume minimisation, Comm. Math. Phys., 280 (2008), 611-673.

[25] D. Page, A compact rotating gravitational instanton, Phys. Lett. B, 79 (1978), 235-238.

[26] J. Sparks, Sasaki-Einstein manifolds, Surveys in differential geometry, XVI, Geometry of special holonomy and related topics, Surv. Differ. Geom., 16, Int. Press, Somerville, MA, 2011, 265-324.

[27] G. Tian and X.-H. Zhu, A new holomorphic invariant and uniqueness of Kähler-Ricci solitons, Comment. Math. Helv., 77 (2002), 297-325.

[28] X.-J. Wang and X. Zhu, Kähler-Ricci solitons on toric manifolds with positive first Chern class, Adv. Math., 188 (2004), 87-103.

\section{Akito FUTAKI}

Graduate School of Mathematical Sciences

The University of Tokyo

3-8-1 Komaba, Meguro-ku

Tokyo 153-8914, Japan

E-mail: afutaki@ms.u-tokyo.ac.jp

Current address:

Yau Mathematical Sciences Center

Tsinghua University

Hai Dian District

Beijing 100084, P.R.China

E-mail: akito@math.tsinghua.edu.cn

\section{Hajime ONO}

Department of Mathematics

Saitama University

255 Shimo-Okubo, Sakura-Ku

Saitama 380-8570, Japan

E-mail: hono@rimath.saitama-u.ac.jp 